\title{
Torque undergone by assemblies of single-domain magnetic nanoparticles submitted to a rotating magnetic field
}

\author{
J. Carrey ${ }^{1, *}$ and N. Hallali ${ }^{1,2}$ \\ ${ }^{1}$ Université de Toulouse, INSA, UPS, Laboratoire de Physique et Chimie des Nano-Objets (LPCNO), \\ 135 avenue de Rangueil, F-31077 Toulouse, France \\ and CNRS, UMR 5215, LPCNO, F-31077 Toulouse, France \\ ${ }^{2}$ CEA Tech LRMP, INSA, Bâtiment 8, 135 avenue de Rangueil, F-31077 Toulouse, France
}

(Received 8 July 2016; revised manuscript received 16 September 2016; published 17 November 2016)

\begin{abstract}
In the last 10 years, it has been shown in various types of experiments that it is possible to induce biological effects in cells using the torque generated by magnetic nanoparticles submitted to an alternating or a rotating magnetic field. In biological systems, particles are generally found under the form of assemblies because they accumulate at the cell membrane, are internalized inside lysosomes, or are synthesized under the form of beads containing several particles. The torque undergone by assemblies of single-domain magnetic nanoparticles has not been addressed theoretically so far and is the subject of the present article. The results shown in the present article have been obtained using kinetic Monte Carlo simulations, in which thermal activation is taken into account, so the torque undergone by ferromagnetic and superparamagnetic nanoparticles could both be simulated. The first system under study is a single ferromagnetic particle with its easy axis in the plane of the rotating magnetic field. Then, elements adding complexity to the problem are introduced progressively and the properties of the resulting system presented and analyzed: random anisotropy axes, thermal activation, assemblies, and finally magnetic interactions. The most complex studied systems are particularly relevant for applications and are assemblies of interacting superparamagnetic nanoparticles with randomly oriented anisotropy axes. Whenever it is possible, analytical equations describing the torque properties are provided, as well as their domain of validity. Although the properties of an assembly naturally derive from those of single particles, it is shown here that several of them were unexpected and are particularly interesting with regard to the maximization of torque amplitude in biological applications. In particular, it is shown that, in a given range of parameters, the torque of an assembly increases dramatically in the direction perpendicular to the plane of the rotating magnetic field. This effect results from a breaking of time reversal symmetry when the field is rotated and is comprehensively explained. This strong enhancement occurs only if the magnetic field rotates, not if it oscillates. When this enhancement does not occur, the total torque of an assembly scales with the square root of the number of particles in the assembly. In the enhancement regime, the total torque scales with a power exponent larger than $1 / 2$. It is also found that, in superparamagnetic nanoparticles, this enhancement is induced by the presence of magnetic interactions so that, in a rather large range of parameters, interacting superparamagnetic particles display a much larger torque than otherwise identical ferromagnetic particles. In all cases studied, the conditions required to obtain this enhancement are provided. The concepts presented in this article should help chemists and biologists in synthesizing nano-objects with optimized torque properties. For physicists, it would be interesting to test experimentally the results described in this article. For this purpose, torque measurements on well-characterized assemblies of nanoparticles should be performed and compared with numerical simulations.
\end{abstract}

DOI: 10.1103/PhysRevB.94.184420

\section{INTRODUCTION}

Magnetic nanoparticles (MNPs) have several properties which can be used in biology [1,2]. Submitted to a highfrequency magnetic field, MNPs release heat-the so-called magnetic hyperthermia-which can be used to destroy cancer cells [3], release drugs [4], and activate gene expression [5], neurons [6] or other biological processes. When placed in a magnetic gradient, they generate a force which can be used to enhance calcium influx inside cells, to improve transfection rates for gene therapy, stem cell differentiation, and tissue engineering [7]. This force can also be used to manipulate molecules, to stimulate the growth of bone cells or neurons $[1,8]$, to drive drug delivery [9], or to extract molecules captured by MNPs in bioseparation processes.

\footnotetext{
*julian.carrey@insa-toulouse.fr
}

When placed in a homogeneous magnetic field, under certain conditions, MNPs also experience a torque, which is for instance used to twist molecules [10], degrade proteins, activate enzymes, trigger chemical reactions, or activate cell growth [1,2,11-14]. Recently, it has also been observed that it was possible to induce the death of cancer cells using MNPs internalized inside lysosomes using a small-amplitude (20 mT), low-frequency rotating magnetic field [15]. It is not unreasonable to expect in the future triggering a large variety of biological signals using this principle [1,2].

Calculating the torque undergone by MNPs submitted to a magnetic field is not only useful to interpret quantitatively such experiments but should also permit prediction of the parameters maximizing the torque, which is especially useful when the final objective is to destroy cancer cells. To be relevant for biological applications, calculations should be performed on assemblies of MNPs. Indeed, in biological systems, one rarely faces isolated objects: MNPs have a strong 
tendency to form aggregates at the surface of cell membranes; when internalized inside cells, they often accumulate inside lysosomes, where they are generally well packed; finally, commercial or laboratory-synthesized MNPs are sometimes obtained in the form of beads containing several MNPs.

Several theoretical papers of interest have addressed the torque, displacement, and hysteresis loops of magnetic objects inside an alternating or rotating magnetic field [16-22]. References [18-20]-motivated by an application in magnetic hyperthermia-were more focused on the shape and area of the hysteresis loop than on the torque amplitude. In Ref. [21], the authors calculated the torque undergone by magnetic microdisks presenting a vortex state. In Ref. [22], the rotation speed of a MNP in a fluid was calculated using linear response theory. Finally, in Ref. [17], M. M. van Oene et al. have used an approach similar to the one used here. They provide complete results of the torque undergone by MNPs using several models, and in particular that of Stoner-Wohlfarth, which is very appropriate for describing MNPs. However, when dealing with an assembly of $N$ MNPs, the authors hypothesize that all the anisotropy axes of the MNPs are parallel. In this case, the torque of the assembly is simply $N$ times the torque of a single MNP. However, in real systems, this hypothesis is rarely verified, so the case of anisotropy axis randomly oriented in space should be studied to be applicable to biology experiments.

One could a priori think that this additional hypothesis should not change the problem dramatically and that the final results would be approximately the same as with parallel axes, except that the total torque would simply be multiplied by a given constant factor. The objective of this article is to show that this is not the case, and that the results obtained for an assembly of MNPs with randomly oriented axes are very different from the ones obtained for single MNPs. For instance, for single MNPs, maximizing the torque simply requires maximizing the anisotropy and/or the magnetic field. In an assembly of MNPs, it will be shown that an anisotropy or magnetic field that is too large decreases the torque. Also, it will be shown that, in certain conditions, assemblies of superparamagnetic NPs generate a larger torque than ferromagnetic ones, which is never the case for isolated MNPs. Although a few results, which will be presented below, could appear counterintuitive at first sight, it will be shown that most of them can be understood qualitatively and sometimes quantitatively.

To perform these theoretical studies, a home-made kinetic Monte Carlo program was used. The main interest of this algorithm is that it takes into account thermal activation, so that MNPs in the ferromagnetic and superparamagnetic regime can be simulated indifferently without any special assumption. This program has proven to be very useful in magnetic hyperthermia for theoretical studies on the heating power of assemblies of interacting MNPs and in interpreting experimental results $[23,24]$. The second interest of this program is the implementation of magnetic interactions, which will also be addressed in this article, because it is crucial in assemblies of MNPs.

This article is organized as follows. The simulations will be presented briefly, and various systems will be studied, starting from single ferromagnetic MNPs with their easy axes in the plane of the rotating field. Then, elements adding complexity will be introduced progressively: random axes, thermal activation, assembly of particles, and magnetic interactions. Finally, given the lengthiness of this article, a summary of the main results will be provided before concluding.

\section{BASIC THEORY AND SIMULATIONS USED}

The magnetic properties of the MNP assembly have been calculated using a kinetic Monte Carlo program, which has been described in detail in Ref. [24]. The only modifications made to the software described in that reference are: (i) the possibility of rotating a constant norm magnetic field inside a plane and (ii) calculation of the $X Y Z$ components of the magnetization so that the $X Y Z$ components of the torque can also be calculated. A brief summary of the key ingredients of the simulations are presented thereafter.

This paper is restricted to the case of immobilized singledomain MNPs. Perfectly monodisperse MNPs of diameter $d$ and volume $V$, with a uniaxial anisotropy $K_{\text {eff }}$ and displaying a magnetization per unit volume $M_{S}$, are considered. We will also always remain in the two-level approximationthe magnetization can only have two possible positions-so the excited states into a potential well are not taken into consideration. With these approximations, the energy of a MNP is given by

$$
E(\theta, \phi)=K_{\mathrm{eff}} V \sin ^{2}(\theta)-\mu_{0} M_{S} V H_{\mathrm{tot}} \cos (\theta-\phi),
$$

where $\mu_{0} H_{\text {tot }}$ is the norm of the magnetic field $\overrightarrow{\mu_{0} H_{\text {tot }}}$ acting on the MNP, $\theta$ is the angle between the easy axis and magnetization, and $\phi$ is the angle between the easy axis and $\overrightarrow{\mu_{0} H_{\text {tot }}}$ [see Fig. 1(a)].

In most cases studied here, an external rotating magnetic field $\overrightarrow{\mu_{0} H_{\text {ext }}}$ of norm $\mu_{0} H_{\max }$ and frequency $f$ is applied to the MNP assembly. In this case, if the magnetic field rotates in the, say, $X Y$ plane, the $X$ and $Y$ components of $\overrightarrow{\mu_{0} H_{\text {ext }}}$ are given by, respectively,

$$
\mu_{0} H_{\mathrm{ext}}^{X}=\mu_{0} H_{\max } \cos (2 \pi f t)
$$

and

$$
\mu_{0} H_{\mathrm{ext}}^{Y}=\mu_{0} H_{\max } \sin (2 \pi f t) .
$$

In one restricted part of the article, the case of an oscillating magnetic field will be studied. In this case, if the magnetic field is applied in the, say, $X$ direction, the $X$ component of $\overrightarrow{\mu_{0} H_{\text {ext }}}$ is given by

$$
\mu_{0} H_{\mathrm{ext}}^{X}=\mu_{0} H_{\mathrm{max}} \cos (2 \pi f t),
$$

and the $Y$ component is null.

In the article, the dipolar interactions between MNPs will sometimes be neglected and sometimes taken into account. In the former case, $\overrightarrow{\mu_{0} H_{\mathrm{tot}}}=\overrightarrow{\mu_{0} H_{\mathrm{ext}}}$. In the latter case, $\overrightarrow{\mu_{0} H_{\mathrm{tot}}}$ is the sum of $\overrightarrow{\mu_{0} H_{\text {ext }}}$ and of the dipolar field $\overrightarrow{\mu_{0} H_{\text {dip }}}$ created by the other NPs. The latter is calculated using

$$
\overrightarrow{\mu_{0} H_{\mathrm{dip}}}=\frac{\mu_{0} M_{S} V}{4 \pi} \sum_{i \neq j} \frac{3\left(\overrightarrow{m_{j}} \cdot \overrightarrow{e_{i j}}\right) \overrightarrow{e_{i j}}-\overrightarrow{m_{j}}}{r_{i j}^{3}},
$$

where $\overrightarrow{e_{i j}}$ is the unitary vector joining two NPs, $\vec{m}_{j}$ is the unitary vector linked to the magnetization orientation, and 


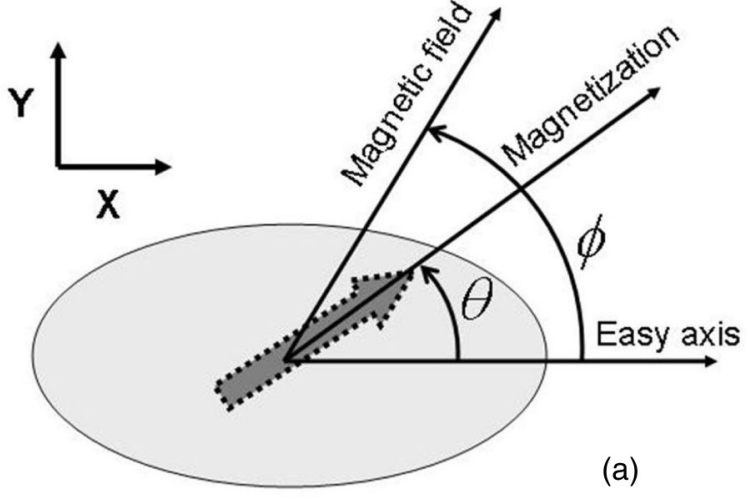

(a)

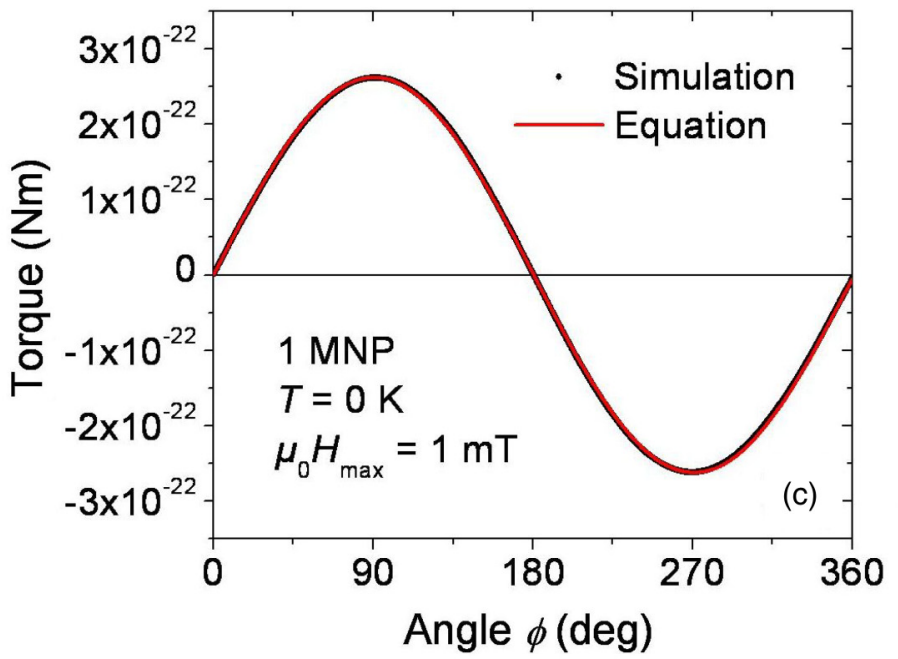

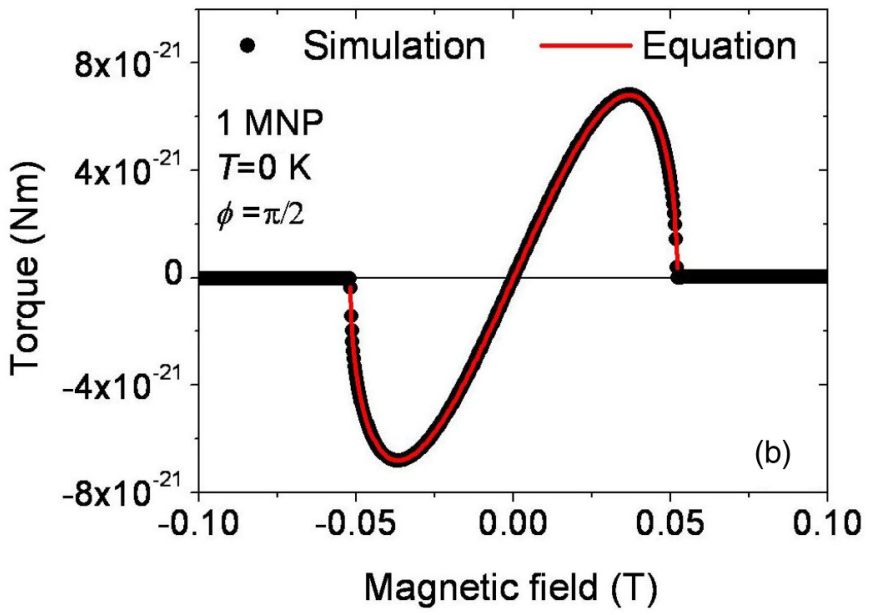

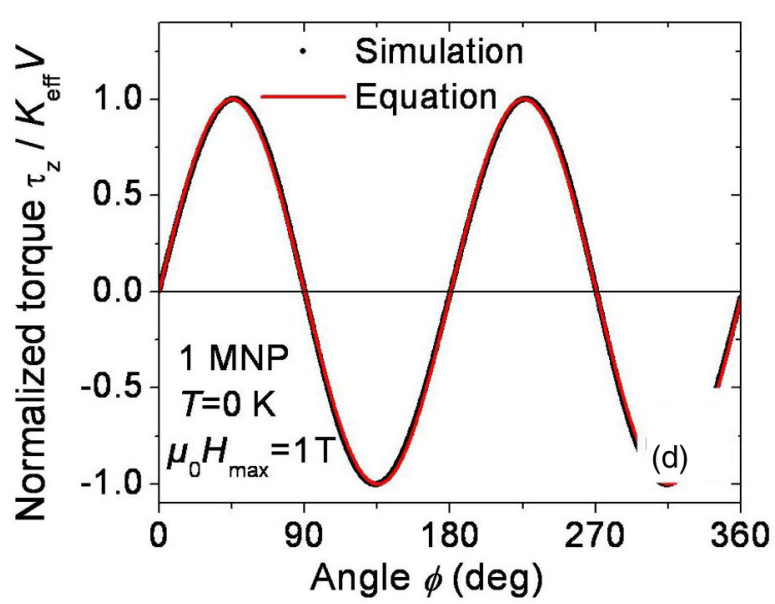

FIG. 1. (a) Schematic of a NP illustrating the angles and axes used in the main text. (b) Torque undergone by a single ferromagnetic NP $(T=0 \mathrm{~K})$ submitted to an alternating magnetic field with $\phi=\frac{\pi}{2}$. Dots are simulations; line is calculated using Eq. (7). (c, d) Torque undergone by a single ferromagnetic NP $(T=0 \mathrm{~K})$ submitted to a rotating field of (c) $1 \mathrm{mT}$ and (d) $1 \mathrm{~T}$. Dots are simulations. Line is calculated using (c) Eq. (9) and (d) Eq. (10).

$r_{i j}$ the distance between two NPs. This sum is calculated exactly without Ewald summation or a cutoff radius.

Using the algorithm described in Ref. [24], the magnetization of each MNP is calculated as a function of time. The algorithm takes into account thermal activation so that thermally activated jumps between the two energy minima over the energy barrier are allowed and taken into account when temperature $T>0$. Once the $X Y Z$ components of magnetization for each particle have been calculated, the total magnetic moment of the assembly $\vec{m}$ is calculated by performing a vector addition. Finally, the torque $\vec{\tau}$ undergone by the MNP assembly is calculated using

$$
\vec{\tau}=\mu_{0} \vec{m} \times \overrightarrow{H_{\mathrm{ext}}}
$$

In all the results shown in this article, when calculating the torque undergone by MNPs under the influence of a rotating magnetic field, a full rotation of the magnetic field is first performed and the results laid aside because a transient regime occurs; only the second rotation, during which a constant regime is achieved, is recorded and treated. The reason for the presence of this transient regime will be clarified later and illustrated in Sec. IV B.

Unless otherwise specified, the parameters used in the simulations are $K_{\text {eff }}=13 \mathrm{~kJ} / \mathrm{m}^{3}, \mu_{0} H_{\max }=10 \mathrm{mT}, M_{S}=0.5 \times$ $10^{6} \mathrm{~A} / \mathrm{m}, d=10 \mathrm{~nm}, T=300 \mathrm{~K}$, and the jump attempt frequency over the energy barrier $v_{0}=10^{10} \mathrm{~Hz}$. The magnetic parameters are thus those of magnetite, a widely used material in biomedical applications.

\section{TORQUE UNDERGONE BY A SINGLE PARTICLE}

Here, the torque undergone by a single particle will be presented for a few cases of interest. The objectives are threefold: (i) to present a few cases where simple analytical formulas for the torque are valid, (ii) to illustrate that, in these cases, there is perfect agreement between the simulations and the analytical formulas, which is a way to validate our program, and (iii) to understand the physics behind this phenomenon, starting with the simple case; it will be shown that, even in simple cases, some nontrivial effects are sometimes encountered. 


\section{A. A single ferromagnetic particle with its easy axis in the plane of the field}

The case of a purely ferromagnetic particle is presented. For that purpose, the temperature is set to $T=0 \mathrm{~K}$. Since there are no thermal fluctuations in this case, the magnetization never jumps over the energy barrier and stays inside the local or global energy minimum. The torque can be calculated analytically in the case where an alternating magnetic field is applied perpendicularly to the easy axis of the MNP, i.e., with $\phi=\frac{\pi}{2}$. In this case, the equilibrium position of magnetization is found by searching the $\theta$ value for which $\frac{\partial E}{\partial \theta}=0$, leading to $\sin \theta=\frac{\mu_{0} M_{S} H_{\text {tot }}}{2 K_{\text {eff }}}$, and using Eq. (6),

$$
\tau_{Z}=\tau_{\max } \sqrt{1-\left(\frac{\mu_{0} M_{S} H_{\mathrm{tot}}}{2 K_{\mathrm{eff}}}\right)^{2}}
$$

with

$$
\tau_{\max }=\mu_{0} M_{S} V H_{\max } .
$$

The comparison between Eq. (7) and simulations is shown in Fig. 1(b), with $\mu_{0} H_{\max }=50 \mathrm{mT}$. The torque displays a maximum since, for a large applied magnetic field, the magnetization and magnetic field are aligned, cancelling the torque. A perfect agreement between Eq. (7) and simulations is found.

Another simple case is when a MNP is submitted to a magnetic field (i) rotating in a plane including the MNP easy axis and (ii) small enough so the particle magnetization does not rotate under its influence $(\theta=0)$. In this case, the torque is simply given by

$$
\tau_{Z}=\tau_{\max } \sin \phi,
$$

assuming that the easy axis is along the $X$ axis defined in Fig. 1(a) and that the field rotates in the $(X Y)$ plane. The comparison between simulations and Eq. (9) is shown in Fig. 1(c). A magnetic field of $1 \mathrm{mT}$, much smaller than the anisotropy field (see below), was used, so the magnetization stayed along the $X$ axis during the magnetic field rotation. With these conditions, a perfect agreement between simulations and Eq. (9) was found. Thus, Eq. (9) is valid only if the anisotropy of the particle is large enough and/or the magnetic field small enough (see below).

Finally, it was shown in Ref. [17] that, for a very large applied magnetic field, the torque equals

$$
\tau_{Z}=K_{\text {eff }} V \sin 2 \phi .
$$

In Fig. 1(d), the comparison between this equation and simulations for a magnetic field of $1 \mathrm{~T}$ is shown. Here again, perfect agreement is found.

Equations (9) and (10) are two limit cases corresponding to a low and high magnetic field, respectively; the intermediate cases must be calculated numerically. To do so, the torque as a function of $\phi$ has been calculated for a fixed value of $K_{\text {eff }}=$ $13 \mathrm{~kJ} / \mathrm{m}^{3}$ and various values of $\mu_{0} H_{\max }$. Data for $\mu_{0} H_{\max }$ in the range $1 \mathrm{mT}-1 \mathrm{~T}$ are shown in Figs. 2(a)-2(c). Figures 2(a) and 2(b) illustrate the evolution of the magnetization angle $\theta$ when the magnetic field rotates, and Fig. 2(c) shows the corresponding torque amplitude. In Fig. 2(c), values of the ratio $\frac{\mu_{0} H_{\max }}{\mu_{0} H_{K}}$ are also provided, where $\mu_{0} H_{K}$ is the anisotropy
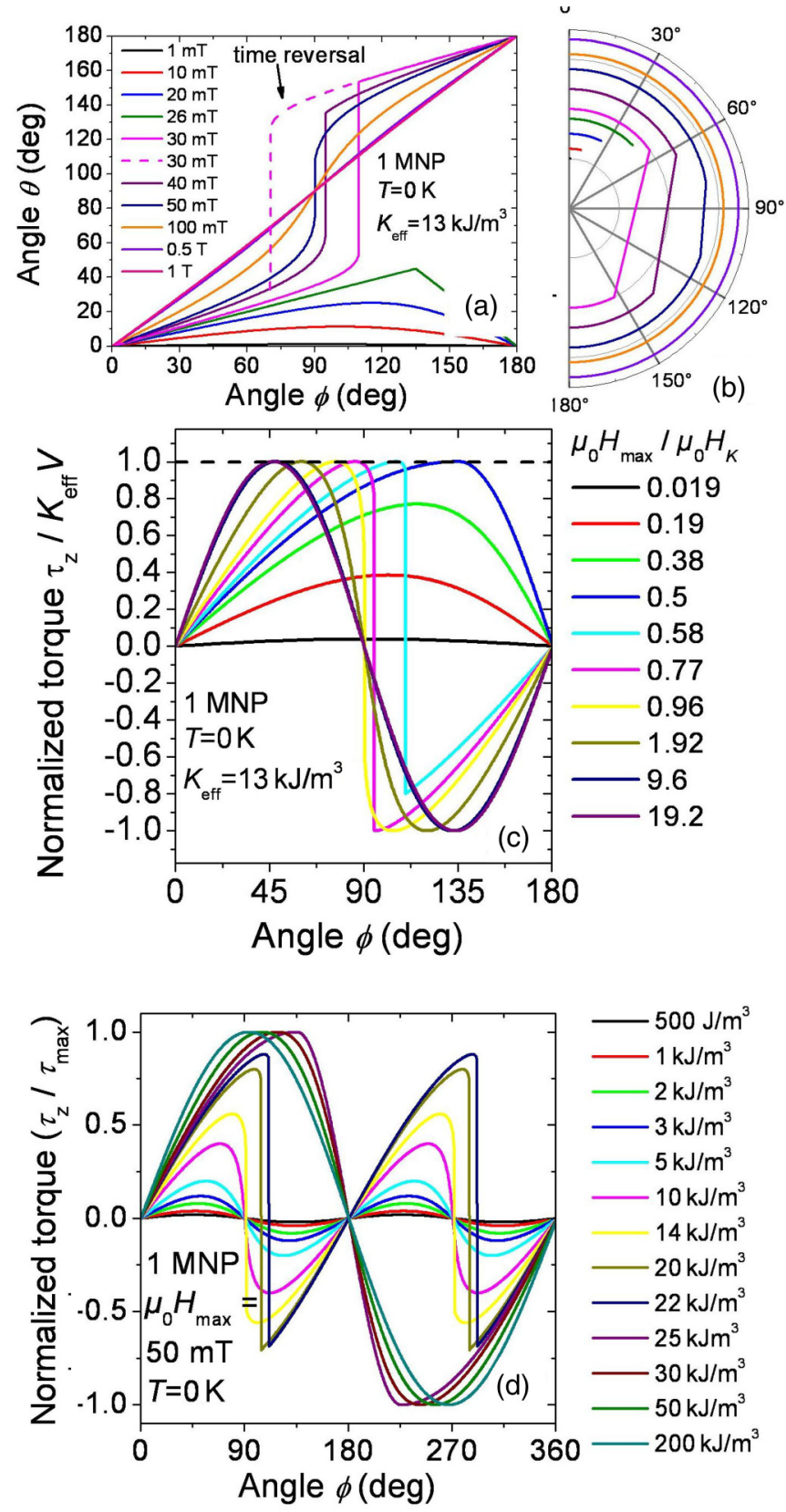

FIG. 2. (a-c) A single ferromagnetic $\mathrm{NP}(T=0 \mathrm{~K})$ is submitted to a rotating magnetic field varying between $1 \mathrm{mT}$ and $1 \mathrm{~T}$. Its easy axis is in the plane of the field. $K_{\text {eff }}=13 \mathrm{~kJ} / \mathrm{m}^{3}, d=10 \mathrm{~nm}$. (a) Evolution of $\theta$ as a function of $\phi$. Initially, magnetization is at $\theta=0$. The dashed pink line illustrates a rotation of the field in the opposite direction for $\mu_{0} H_{\max }=30 \mathrm{mT}$. (b) Trajectory of $\theta$ for various values of $\mu_{0} H_{\max }$. (c) Normalized torque $\frac{\tau_{Z}}{K_{\text {eff }} V}$ as a function of $\phi$. Normalized magnetic field values $\frac{\mu_{0} H_{\max }}{\mu_{0} H_{K}}$ are given in the legend for information, but raw magnetic field values are the same as in (a). (d) Normalized torque $\frac{\tau_{Z}}{\tau_{\max }}$ for a single ferromagnetic NP $(T=0 \mathrm{~K})$ submitted to a rotating field of $50 \mathrm{mT}$ with an anisotropy $K_{\text {eff }}$ varying between $500 \mathrm{~J} / \mathrm{m}^{3}$ and $200 \mathrm{~kJ} / \mathrm{m}^{3}$. Its easy axis is in the plane of the field.

field defined as $\mu_{0} H_{K}=\frac{2 K_{\text {eff }}}{M_{S}}$. For parameter values used in Fig. $2, \mu_{0} H_{K}=52 \mathrm{mT}$. Similarly, the evolution of the torque for a fixed value of $\mu_{0} H_{\max }=50 \mathrm{mT}$ and for $K_{\text {eff }}$ varying 
between $500 \mathrm{~J} / \mathrm{m}^{3}$ and $200 \mathrm{~kJ} / \mathrm{m}^{3}$ is shown in Fig. 2(d). A careful analysis of Figs. 2(c) and 2(d) permits extraction of the domain of validity of Eqs. (9) and (10). Equation (9) is valid at the condition $\mu_{0} H_{\max }<0.06 \mu_{0} H_{K}$. When this condition is fulfilled, the magnetization of the MNPs can be considered fixed and is not influenced by the presence of the rotating magnetic field. Equation (10) is valid at the condition $\mu_{0} H_{\max }>12 \mu_{0} H_{K}$.

The angular dependence of the torque shown in Figs. 2(a) and 2(b) presents several features, which will be of importance in understanding the properties of MNP assemblies discussed below. First, at low magnetic field, when $\mu_{0} H_{\max } \leqslant$ $\frac{\mu_{0} H_{K}}{2}$, the magnetization rotates partially with the rotating magnetic field, but eventually comes back smoothly to its initial position [see Fig 2(a)]. When $\mu_{0} H_{\max } \geqslant \mu_{0} H_{K}$, the magnetization performs a continuous rotation, approximately following the magnetic field. For $\mu_{0} H_{\max } \gg \mu_{0} H_{K}, \theta \approx \phi$. As will be clearer below, the most interesting regime occurs when $\frac{\mu_{0} H_{K}}{2}<\mu_{0} H_{\max }<\mu_{0} H_{K}$. This regime comes with three features: (i) Magnetization switches during the magnetic field rotation, going abruptly to a second equilibrium position. This switch is associated with an abrupt change in the sign of the torque [see Figs. 2(c) and 2(d)]. (ii) As previously noted and illustrated in Ref. [17], there is a breaking of time reversal symmetry: magnetic properties are not the same if the magnetic field rotates clockwise or counterclockwise. This is illustrated in Fig. 2(a) for $\mu_{0} H_{\max }=30 \mathrm{mT}$. (iii) The $\tau_{Z}(\phi)$ function is not symmetrical with respect to the abscissa axis, so the mean value of $\tau_{Z}$ is nonzero during one full rotation. This can be seen clearly for $\frac{\mu_{0} H_{\max }}{\mu_{0} H_{K}}=0.58$ in Fig. 2(c) or for $K_{\text {eff }}=22 \mathrm{~kJ} / \mathrm{m}^{3}$ in Fig. 2(d). This latter point is important, and its consequences will be discussed again when studying the properties of assemblies.

From Figs. 2(c) and 2(d), it is possible to extract the maximum value of the torque as a function of the anisotropy value for a constant magnetic field or reciprocally. Such data are displayed in Fig. 3. Figure 3(a) shows the maximum torque obtained during the rotation of the magnetic field as a function of $\mu_{0} H_{\max }$. The two features of interest are (i) the maximum torque $\tau=K_{\text {eff }} V$ is reached for $\mu_{0} H_{\max }=\frac{K_{\text {eff }}}{M_{S}}$ and (ii) below the saturation, the maximum torque that a MNP can undergo is linear with the magnetic field and is given by

$$
\tau=\mu_{0} H_{\max } M_{S} V=\tau_{\max } .
$$

Similarly, Fig. 3(b) shows the maximum torque obtained during the rotation of the magnetic field as a function of $K_{\text {eff }}$ for a constant $\mu_{0} H_{\max }$. Two features of interest are evidenced: (i) $\tau_{\max }$ is reached when $K_{\text {eff }}=\mu_{0} H_{\max } M_{S}$ and (ii) below saturation, the maximum torque that a MNP can undergo is linear with its anisotropy. It is thus given by

$$
\tau=\tau_{\max } \frac{K_{\text {eff }}}{\mu_{0} H_{\max } M_{S}}=K_{\text {eff }} V .
$$

The data in Figs. 3(a) and 3(b) can thus be summarized quite simply. The optimal matching between anisotropy and magnetic field occurs when $K_{\text {eff }}=\mu_{0} H_{\max } M_{S}$. If the anisotropy is too small compared with the magnetic field, the
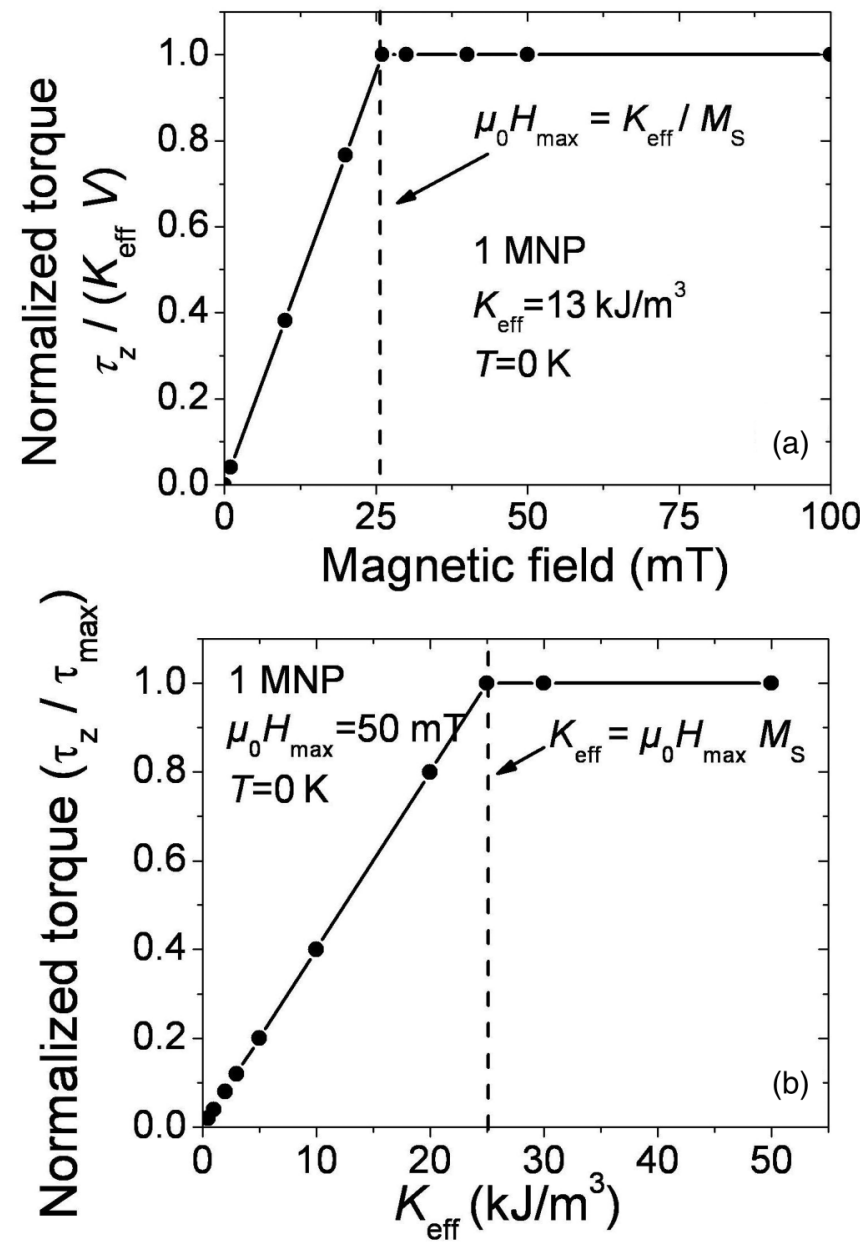

FIG. 3. (a, b) The maximum value of torque obtained in Figs. 2(c) and 2(d) are plotted as a function of (a) $\mu_{0} H_{\max }$ for a constant $K_{\text {eff }}=$ $13 \mathrm{~kJ} / \mathrm{m}^{3}$ (b) $K_{\text {eff }}$ for a constant $\mu_{0} H_{\max }=50 \mathrm{mT}$. In both graphs, the vertical line displays the condition for which $K_{\mathrm{eff}}=\mu_{0} H_{\max } M_{S}$.

torque is limited to $K_{\text {eff }} V$; if the magnetic field is too small, the torque is limited to $\tau_{\max }$.

\section{B. A single ferromagnetic particle with randomly oriented axis}

The case where a single ferromagnetic NP $(T=0 \mathrm{~K})$ has its easy axis randomly oriented in space is now studied. To perform our calculation, the total torque $|\tau|$ undergone by a particle submitted to a $50 \mathrm{mT}$ rotating magnetic field was calculated 25 times. We will hereafter call a "cycle" one full rotation of the magnetic field. For each new cycle, a different easy axis, randomly oriented in space, was drawn. Figure 4(a) shows as an example 12 cycles calculated for $K_{\text {eff }}=500 \mathrm{~J} / \mathrm{m}^{3}$. It can be observed that, for some cycles, the maximum theoretical torque $K_{\text {eff }} V$ is reached, but not for others. For each cycle, the maximum value of the torque was determined, and then the mean value and standard deviation of this maximum was calculated over 25 cycles. These data are plotted in Fig. 4(b). It can be observed that these data resemble those in Fig. 3(b), which also have been plotted in Fig. 4(b) for comparison. However, since $K_{\text {eff }} V$ is not reached for each value, the averaged value of the maximum torque is slightly smaller. For a better visualization of these data, 

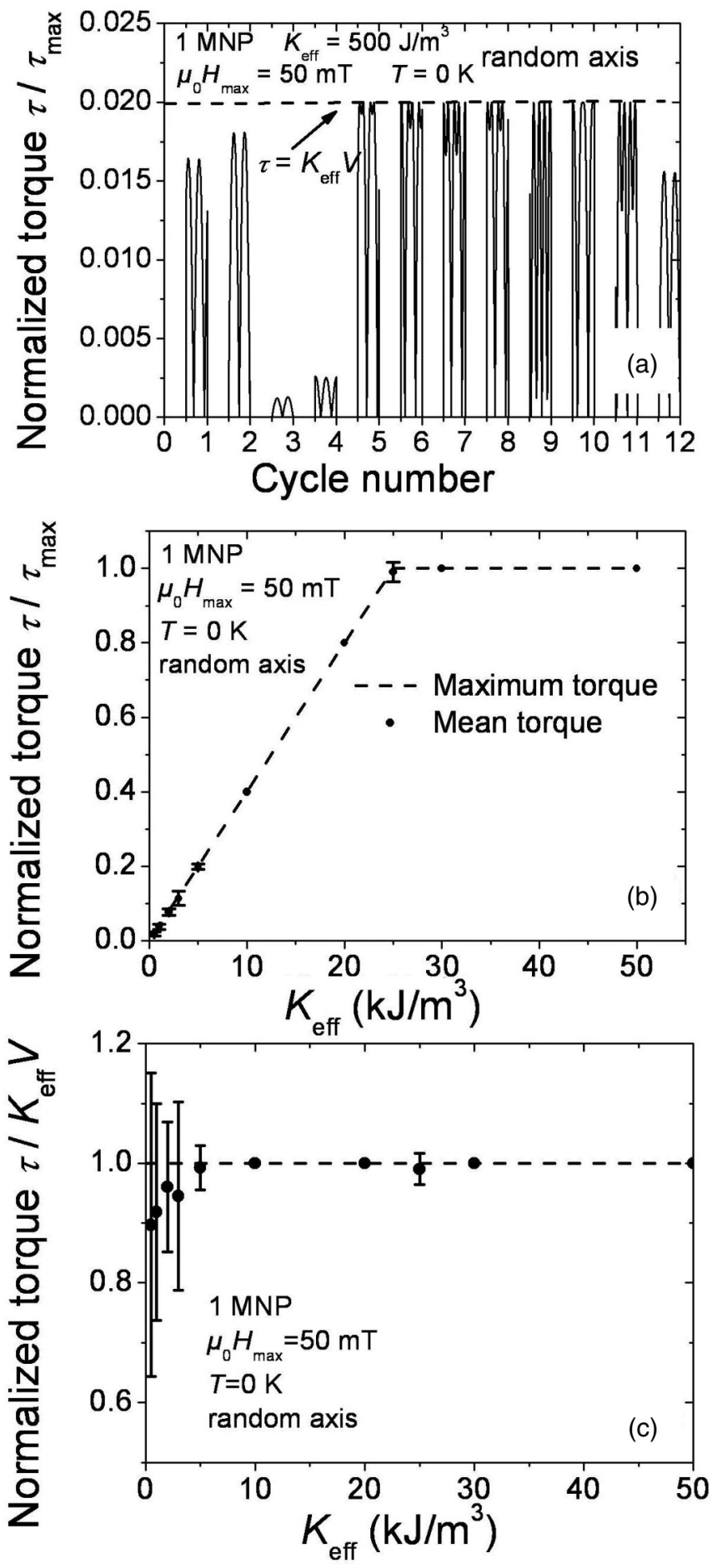

FIG. 4. (a) Normalized torque $\frac{|\tau|}{\tau_{\max }}$ for a single ferromagnetic $\mathrm{NP}(T=0 \mathrm{~K})$ submitted to a rotating field of $50 \mathrm{mT}$ with $K_{\text {eff }}=$ $500 \mathrm{~J} / \mathrm{m}^{3} \cdot \frac{|\tau|}{\tau_{\max }}$ as a function of time is shown for 12 different easy axis configurations. Each configuration (referred as "Cycle number") corresponds to a new random orientation of the easy axis and to a $360^{\circ}$ rotation of the magnetic field. Each cycle is separated from the other by a blank space, for clarity. The horizontal dashed line indicates $\tau=$ $K_{\text {eff }} V$. (b) Dots are the mean value and standard deviation, averaged over 25 cycles, of the maximum torque reached in data similar to (a). The line displays the data of Fig. 3(b). (c) Same data as (b) except that $\frac{|\tau|}{K_{\text {eff }} V}$ is plotted.

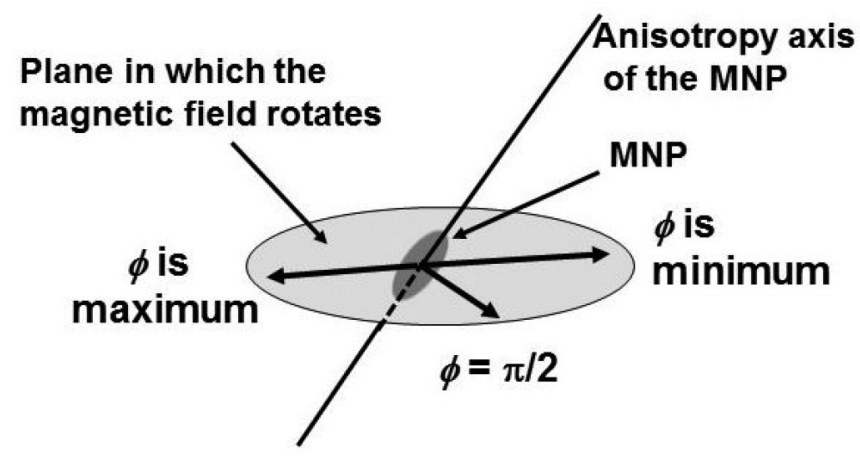

FIG. 5. Illustration of a MNP (shown as a small dark grey ellipse) with any angle between its easy axis and the plane in which the magnetic field rotates (shown as large light grey disk). The arrows represent various positions of the magnetic field for which $\phi$ is maximum, $\phi$ is minimum, and $\phi=\pi / 2$.

Fig. 4(c) shows the data of Fig. 4(b) normalized by $K_{\text {eff }} V$. It is clear from Fig. 4(c) that the fact $K_{\text {eff }} V$ is not always reached during a cycle has an anisotropy-dependant origin: for anisotropies below $10 \mathrm{~kJ} / \mathrm{m}^{3}$, this is often the case, but this is extremely rare for higher anisotropies, except around $25 \mathrm{~kJ} / \mathrm{m}^{3}$, where it appears again.

As a matter of fact, this phenomenon can be easily understood from simple considerations, illustrated in Fig. 5: when a MNP has a random orientation axis, the angle $\phi$ does not vary between 0 and $2 \pi$ but between a minimum and a maximum value between which $\frac{\pi}{2}$ is always included. In other words, there is always a position of the magnetic field during its rotation for which $\phi=\frac{\pi}{2}$, but this is not true for the other values. The closer a given value is to $\frac{\pi}{2}$, the higher the probability that there is a position of the field for which $\phi$ equals this value. As a consequence, $\phi$ values near 0 or $\pi$ have a low probability. If one now looks carefully at Fig. 2(d), one can see that the angle for which the maximum torque is reached has a complex behavior as a function of the anisotropy, which is clearly at the origin of the data shown in Figs. 4(b) and 4(c). Indeed, at low anisotropy, the angle for which the maximum torque is reached is close to $\frac{\pi}{4}$ and increases progressively [see Fig. 2(d)]. In Fig. 4(c) this is associated with a reduced value of the normalized mean torque at low anisotropy, which increases with anisotropy. When anisotropy is in the range $10-20 \mathrm{~kJ} / \mathrm{m}^{3}$ or is very large, the angle for which the maximum torque is reached is close to $\frac{\pi}{2}$ [see Fig. 2(d)]. In Fig. 4(c), it can be seen that, in these conditions, the maximum torque was indeed always reached during the 25 cycles. Figure 4(c) can thus be satisfactorily explained using Figs. 5 and 2(d).

\section{Torque undergone by a superparamagnetic particle}

\section{Influence of thermal activation on the torque properties}

The case of a superparamagnetic particle is now presented. The temperature is thus $T=300 \mathrm{~K}$ in the next simulations, to introduce thermal activation. First, simulations with the MNP diameter varying between 5 and $50 \mathrm{~nm}$ have been calculated to illustrate the progressive transition from the superparamagnetic to the ferromagnetic regime. A single MNP with its easy axis along the $X$ direction is considered and 

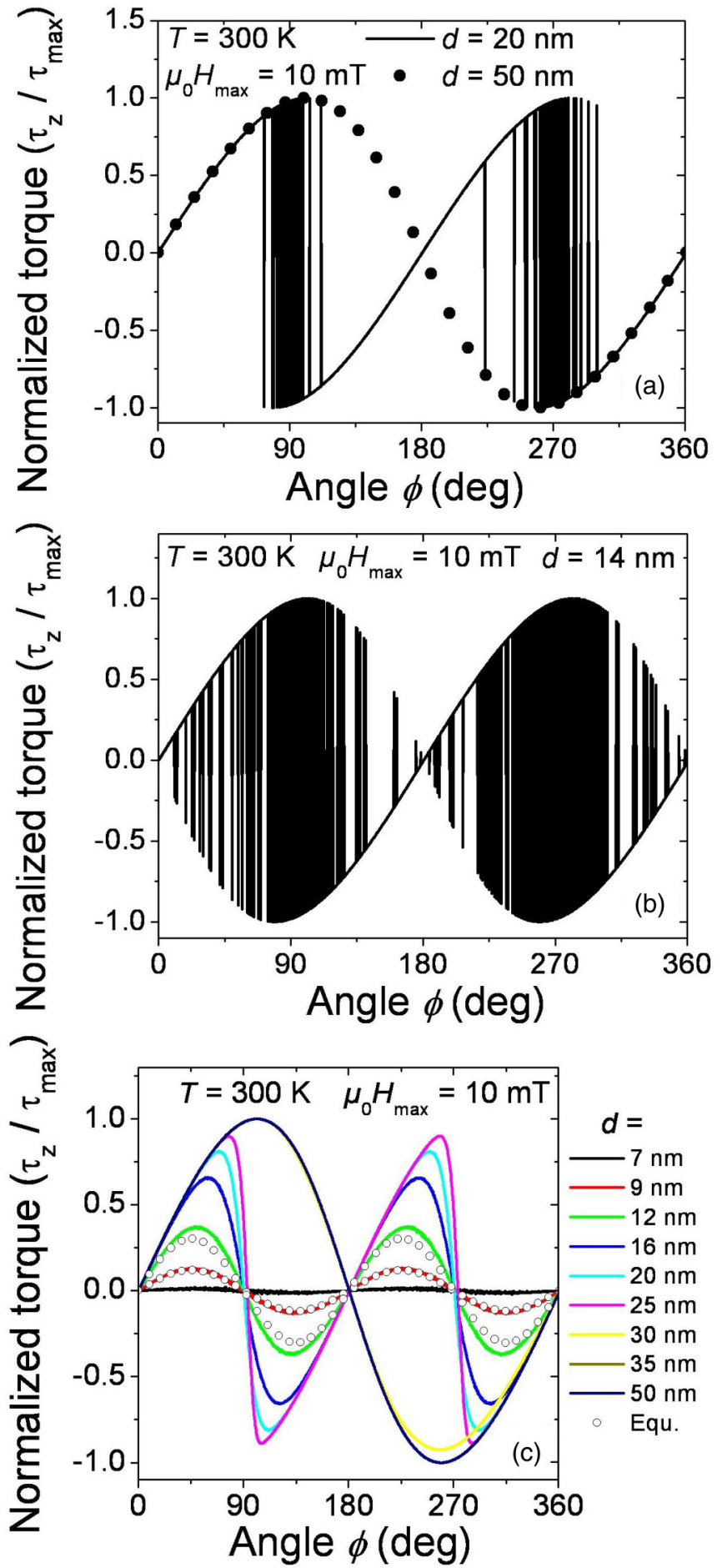

FIG. 6. Study of the superparamagnetic regime, with $T=300 \mathrm{~K}$ and $\mu_{0} H_{\max }=10 \mathrm{mT}$. A single particle is studied with its easy axis along $X$. (a, b) Normalized torque $\frac{\tau_{Z}}{\tau_{\max }}$ as a function of $\phi$ for (a) $d=50$ and $20 \mathrm{~nm}$ and (b) $d=14 \mathrm{~nm}$. (c) Average normalized torque as a function of $d$. Data similar to (a) and (b) have been calculated 50000 times and then averaged. Open dots corresponds to Eq. (18) calculated for $d=9$ and $12 \mathrm{~nm}$.

submitted to a rotating field of $10 \mathrm{mT}$ in the $X Y$ plane. In Figs. 6(a) and 6(b), representative graphs of the torque are shown for $d=50,20$, and $14 \mathrm{~nm}$. For $d$ between 30 and $50 \mathrm{~nm}$, the torque is similar to that observed for a purely ferromagnetic NP $(T=0 \mathrm{~K})$. For $d=25 \mathrm{~nm}$, a few thermally activated jumps between the two equilibrium positions occur (not shown) and become more frequent for $d=20 \mathrm{~nm}$ [see Fig. 6(a)]. Figure 6(a) illustrates clearly that these jumps are more frequent when $\phi$ is close to $\pm \frac{\pi}{2}$ since, when the magnetic field is perpendicular to the easy axis, the magnetic field more efficiently lowers the energy barrier between the two equilibrium positions. When lowering again the diameter of the MNPs, the jumps become more frequent and occur for any $\phi$ value, although they are still more probable when $\phi$ is close to $\pm \frac{\pi}{2}$ [see Fig. 6(b)]. It means that a superparamagnetic particle in a weak external field generates a torque of changing sign at high frequency. This frequency is not the one given by the external magnetic field, but the one given by thermally activated jumps. This frequency is strongly size dependantsimilar to the Néel relaxation time — and is slightly modulated by the rotating external magnetic field because of the angular dependence of the jump probability illustrated in Figs. 6(a) and $6(\mathrm{~b})$.

Thus, using a superparamagnetic particle is a way to apply a high-frequency torque to an entity on which it is bounded without applying a high-frequency magnetic field. On the other hand, it is possible that the entity on which it is bounded-for instance a biological membrane-acts as a low-pass filter so that only the average value of the torque is felt, so its calculation could be useful. Indeed, although it is not easy to see it in Figs. 6(a) and 6(b), the two equilibrium positions do not generate a torque with exact opposite values, and the probabilities of being in one of the two positions are not equal: the position in the direction of the field is always more probable. This means that the average torque is nonzero. In Fig. 6(c), the average value of the torque as a function of $\phi$ and $d$ is provided. It has been calculated by averaging 50000 graphs similar to those shown in Figs. 6(a) and 6(b). It is clear from this figure that the thermal activation strongly decreases the average torque undergone by the MNP. This effect is also enhanced by the fact that $\tau_{\max }$ strongly diminishes with $d$, although this is not illustrated by Fig. 6(c), which displays the normalized torque $\frac{\tau_{Z}}{\tau_{\max }}$ only.

\section{Analytical expression for the average torque}

An analytical expression giving the average torque undergone by a superparamagnetic NP as a function of the angle at low magnetic field has been found. Without loss of generality, let us assume that the MNP easy axis is along $X$. If so, when the magnetic field is aligned along the easy axis $X$, the magnetization along $X$ is given by [25]

$$
M_{X}=M_{S} \tanh \left(\frac{\mu_{0} V H_{\mathrm{ext}} M_{S}}{k_{B} T}\right) .
$$

When the magnetic field is aligned perpendicularly to the easy axis, i.e., along $Y$, it can be derived from Eq. (1) that

$$
M_{Y}=\frac{M_{S}^{2} \mu_{0} H_{\mathrm{ext}}}{2 K_{\mathrm{eff}}}
$$

when $\mu_{0} H_{\text {ext }}<\frac{2 K_{\text {eff }}}{M_{S}}$, and $M_{Y}=M_{S}$ above. We have then hypothesized that, for a small enough magnetic field, the magnetization for any $\phi$ would be a linear combination of 
these two equations, so that

$$
M_{X}=M_{S} \cos \phi \tanh \left(\frac{\mu_{0} V H_{\mathrm{ext}} M_{S}}{k_{B} T}\right)
$$

and

$$
M_{Y}=\sin \phi \frac{M_{S}^{2} \mu_{0} H_{\mathrm{ext}}}{2 K_{\mathrm{eff}}} .
$$

Finally, using

$$
\tau_{Z}=V\left(\mu_{0} H_{\mathrm{ext}}^{Y} M_{X}-\mu_{0} H_{\mathrm{ext}}^{X} M_{Y}\right),
$$

then

$$
\tau_{Z}=\frac{\tau_{\max }}{2} \sin 2 \phi\left(\tanh \left(\frac{\tau_{\max }}{k_{B} T}\right)-\frac{\tau_{\max }}{2 K_{\mathrm{eff}} V}\right) .
$$

This equation is plotted in Fig. 6(c), alongside numerical results, for $d=9 \mathrm{~nm}$ and $d=12 \mathrm{~nm}$. For small diameters, up to $d=9 \mathrm{~nm}$, Eq. (18) is in perfect agreement with numerical results. Discrepancy between both starts to occur for diameters larger than $12 \mathrm{~nm}$ (see below).

\section{Domain of validity of the analytical expression and superparamagnetic-ferromagnetic transition}

To study the domain of validity of Eq. (18), a large number of simulations have been performed, where the average torque as a function of the angle was calculated and compared with Eq. (18). The ratio between Eq. (18) and simulations is plotted in Fig. 7(a). A ratio equal to 1 means that the torque amplitude given by the equation and the simulations is the same. Careful analysis of our data allowed us to find the three conditions which need to be fulfilled to have good agreement between equation and simulation. First, Fig. 7(a) illustrates that the dimensionless parameter $\frac{\mu_{0} H_{\max } M_{S} V}{k_{B} T}$ is important: when data are plotted against it, a lot of data points collapse on the same universal curve, shown in Fig. 7(a). When this parameter value increases, matching between simulation and equation decreases. A closer look to the raw data (not shown) permits differentiation into two regimes: when $\frac{\mu_{0} H_{\max } M_{S} V}{k_{B} T}<1$, simulations show that the torque has still a $\sin (2 \phi)$ dependence but with an increased amplitude compared with the one given by Eq. (18). This means that, in this regime, Eq. (18) can be corrected using the universal curve shown in Fig. 7(a). When $\frac{\mu_{0} H_{\max } M_{S} V}{k_{B} T}>1$, the torque progressively evolves toward a more complex angular dependence, so Eq. (18) should not be used, even after a correction of its amplitude. Concrete illustrations of these principles will be shown below in Sec. III C 4. The second criterion of validity is that $\mu_{0} H_{\max }$ should be small enough compared with the anisotropy field $\mu_{0} H_{K}$. Curves designated by the arrows labeled " 1 " in Fig. 7(a) illustrate that when $\mu_{0} H_{\max }>0.2 \mu_{0} H_{K}$, small discrepancies between equation and simulation start to appear and that, for larger values of the magnetic field, Eq. (18) is not valid at all. Finally, Fig. 7(a) illustrates that, for some values of parameters, the equation/simulation ratio displays an abrupt drop. Such a drop is illustrated by arrow 2 in Fig. 7(a). This drop occurs when the parameters are such that the magnetization never jumps over the energy barrier during the magnetic field rotation. To make it simple, this can be called a superparamagneticferromagnetic transition. This transition is expected to depend

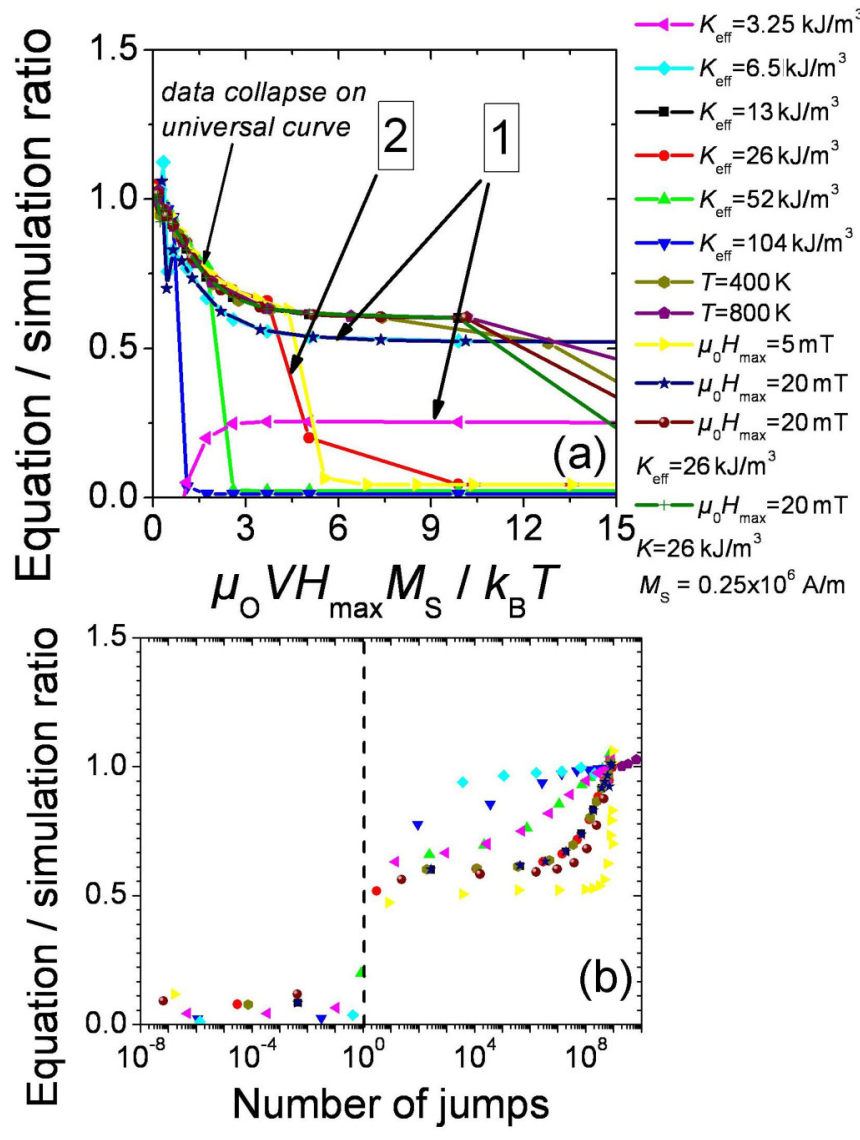

FIG. 7. Study of the validity of Eq. (18). In both graphs the ratio between Eq. (18) and numerical simulations is plotted along the ordinate. When not otherwise specified in the legend, the parameters used in the simulations were $\mu_{0} H=10 \mathrm{mT}, T=300 \mathrm{~K}, K_{\text {eff }}=$ $13 \mathrm{~kJ} / \mathrm{m}^{3}$, and $M_{S}=0.5 \times 10^{6} \mathrm{~A} / \mathrm{m}$. (a) Data are plotted as a function of the dimensionless parameter $\frac{\mu_{0} H_{\max } M_{S} V}{k_{B} T}$. The curves designated by the arrows labeled with a 1 correspond to the cases when $\mu_{0} H<0.2 \mu_{0} H_{K}$ is not satisfied. The jump labeled as 2 can be seen in several other curves and correspond to the transition between the ferromagnetic regime and superparamagnetic regime. (b) The data are plotted as a function of the approximate number of jumps over the energy barrier $n$, provided by Eq. (19). The vertical dashed line shows $n=1$, which corresponds to the transition between a ferromagnetic and a superparamagnetic behavior of the MNP.

on $K_{\text {eff }}, V, M_{S}, \mu_{0} H_{\text {max }}, f$, and $v_{0}$. It was already shown above and illustrated in Figs. 6(a) and 6(b) that the jumps are more probable when $\phi$ is close to $\pm \frac{\pi}{2}$, so this transition is expected to be governed by magnetization switching when $\phi= \pm \frac{\pi}{2}$. It can be easily demonstrated that the jump probability over the barrier in this case equals $v_{0} \exp \left(-\frac{K_{\text {eff }}-\mu_{0} H_{\max } M_{S}}{k_{B} T} V\right)$. The number of jumps $n$ over the energy barrier during one cycle of the magnetic field is thus expected to be roughly given by the equation

$$
n \approx \frac{v_{0} \exp \left(-\frac{K_{\mathrm{eff}}-\kappa \mu_{0} H_{\max } M_{S}}{k_{B} T} V\right)}{f},
$$

where $\kappa=1$. By plotting all the data of Fig. 7(a) as a function of $n$, it is shown that all the drops in the equation/simulation indeed occurs when $n \approx 1$. A good collapse of all data occur 
for $\kappa=1$ (not shown), but a better one is achieved when $\kappa=1.07 \pm 0.04$, especially for data calculated for a low field. Figure 7(b) shows the result of this plotting for $\kappa=1.07$. This means that the last condition of validity of Eq. (18) is that $n>1$ with $\kappa=1.07$.

In summary, when the three conditions $n>1, \mu_{0} H_{\max }<$ $0.2 \mu_{0} H_{K}$, and $\frac{\mu_{0} H_{\max } M_{S} V}{k_{B} T}<1$ are fulfilled, it is possible to calculate without simulations the average torque undergone by a superparamagnetic particle. If $\frac{\mu_{0} H_{\max } M_{S} V}{k_{B} T} \ll 1$, Eq. (18) can be used directly. If $\frac{\mu_{0} H_{\max } M_{S} V}{k_{B} T}<1$, Eq. (18) underestimates slightly the torque amplitude, but the universal curve shown in Fig. 7(a) can be used to correct the amplitude. When these conditions of validity are not fulfilled, numerical simulations should be used to calculate the torque.

\section{A superparamagnetic particle with an arbitrary angle with respect to the field plane}

The previous part presented the special case where the easy axis of the superparamagnetic MNPs is in the same plane as that in which the magnetic field rotates. The analytical results can be generalized to the case of an arbitrary orientation of the easy axis, taking into consideration the following points: (i) as illustrated in the case of a ferromagnetic particle in Fig. 5, when the particle has an arbitrary angle with respect to the field plane, $\phi$ does not evolve sinusoidally between 0 and $2 \pi$ but sinusoidally between two other angles. (ii) The time evolution of $\phi$ can be easily obtained by calculating the scalar product between the unitary vector of the easy axis and the unitary vector of the rotating magnetic field. This scalar product equals $\cos (\phi)$. (iii) Once the time evolution of $\phi$ has been calculated, Eq. (18) permits calculating the torque amplitude, if the conditions discussed above are fulfilled. To get a more accurate value of the torque amplitude, its value can be divided by the equation/simulation ratio found in Fig. 7(a), using $\frac{\mu_{0} H_{\max } M_{S} V}{k_{B} T}$. (iv) One should note that the torque is, in the general case, not oriented along the $Z$ axis, but its direction is given by the vector product between the easy axis and the magnetic field and has a precession-like motion. (v) We emphasize once more that Eq. (18) provides only the value of the torque averaged on thousands of cycles. During one cycle, the torque evolution would resemble the curves shown in Fig. 6(b).

As an illustration, these few principles have been used to calculate analytically the torque undergone by a MNP with an easy axis out of the plane of the rotating magnetic field and
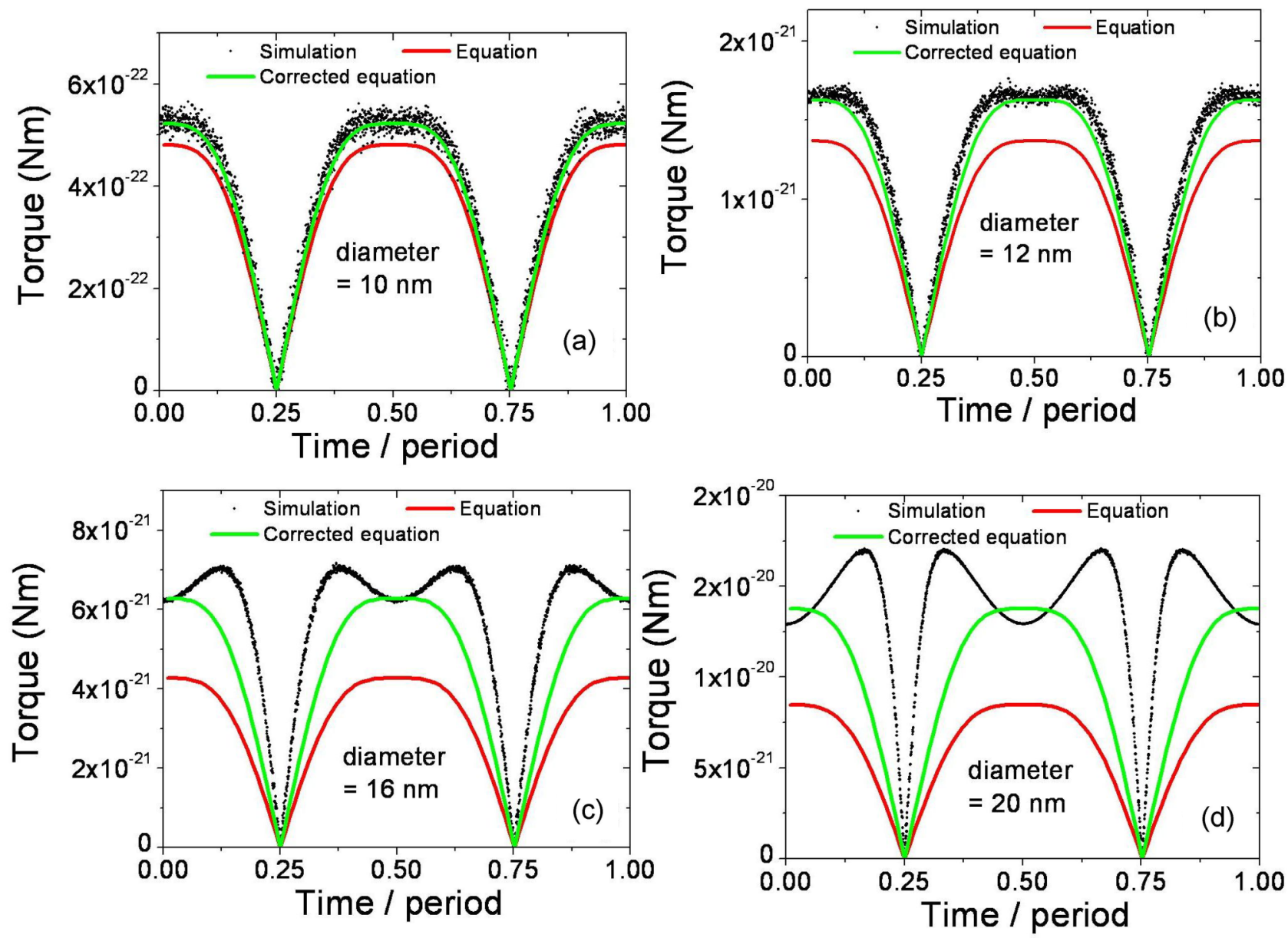

FIG. 8. Torque $|\tau|$ as a function of time for a single superparamagnetic NP, the easy axis of which makes a $\frac{\pi}{4}$ angle with respect to the plane of the rotating magnetic field. The values $d=10,12,16$, and $20 \mathrm{~nm}$ correspond to $\frac{\mu_{0} H_{\max } M_{S} V}{k_{B} T}$ values of approximately $0.6,1.1,2.6$, and 5, respectively. The dots are simulation results; the red line represents Eq. (18); the green line corresponds to Eq. (18) corrected using the universal curve of Fig. 7(a) (see text). 
making a $\frac{\pi}{4}$ angle with respect to this plane. The results are shown in Fig. 8 for MNPs with diameters of 10, 12, 16, and $20 \mathrm{~nm}$, corresponding to $\frac{\mu_{0} H_{\max } M_{S} V}{k_{B} T}$ values of approximately $0.6,1.1,2.6$, and 5. Please note that, in this figure and several others that follow, the absolute value of the torque $|\tau|$-and not $\tau_{Z}$-is plotted. The torque value calculated directly using Eq. (18) and the value corrected using the universal curve in Fig. 7(a) are shown. The agreement is very good up to a diameter of $12 \mathrm{~nm}$. This figure illustrates well that the methods explained above can be used to calculate analytically the torque undergone by a MNP with any angle with respect to the plane in which the field rotates.

\section{TORQUE UNDERGONE BY AN ASSEMBLY OF INDEPENDENT NANOPARTICLES}

Results on the torque generated by an assembly of nanoparticles are now presented. Experimentally, this can be encountered in two cases of interest. The first is in the so-called "beads" of particles, which can be bought in most commercial suppliers of MNPs or are synthesized in several laboratories. They consist in a sphere-generally made of polymers-containing several magnetic particles. Bead size, generally in the range $50-500 \mathrm{~nm}$, is larger or much larger than the size of the MNPs themselves, which is rather in the range $10-20 \mathrm{~nm}$. The second case is what occurs in biological systems when isolated particles are targeted toward cells: they accumulate either at the cell membrane or inside lysosomes, where they form aggregates. The underlying issues are the following: (i) providing that the torque generated by individual MNPs is rather small for reasonable values of MNP volume and anisotropy, is it possible to increase the torque by aggregating several MNPs in beads or at the cell membrane? (ii) If yes, what is the role played by magnetic interactions: are they detrimental or beneficial to the torque amplitude? (iii) What is the best way to maximize the torque? Trying to answer these questions has guided us in the study presented below.

\section{A. Influence of anisotropy and magnetic field amplitude}

The torque undergone by an assembly of $N$ MNPs submitted to a rotating magnetic field is now presented. The case of ferromagnetic and superparamagnetic particles was first studied by stating $T=0 \mathrm{~K}$ and $T=300 \mathrm{~K}$ in simulations with otherwise identical parameters. The case where all the anisotropy axes are aligned in the same direction leads to a trivial result (not shown): the total torque is simply $N$ times the torque undergone by a single particle. The case of randomly oriented anisotropy axes is more complex. Technically, we draw a given random orientation of easy axes, and then calculate the torque as a function of the angle. Then, we draw a new configuration of axes and repeat the calculation. Each time a new configuration is drawn, the obtained $|\tau|(\phi)$ function is different. Representative results for $N=10000, T=0 \mathrm{~K}$, $\mu_{0} H_{\max }=50 \mathrm{mT}$, and various values of $K_{\mathrm{eff}}$ are shown in Fig. 9(a). First, it can be observed that the $|\tau|(\phi)$ function shape depends on the anisotropy: for small anisotropies (500 and $3000 \mathrm{~J} / \mathrm{m}^{3}$ ), the torque is more often a constant value plus a $\sin ^{2}(2 \phi)$-like function. For high anisotropies $\left(30 \mathrm{~kJ} / \mathrm{m}^{3}\right.$ and larger, not shown), it is almost always a constant value
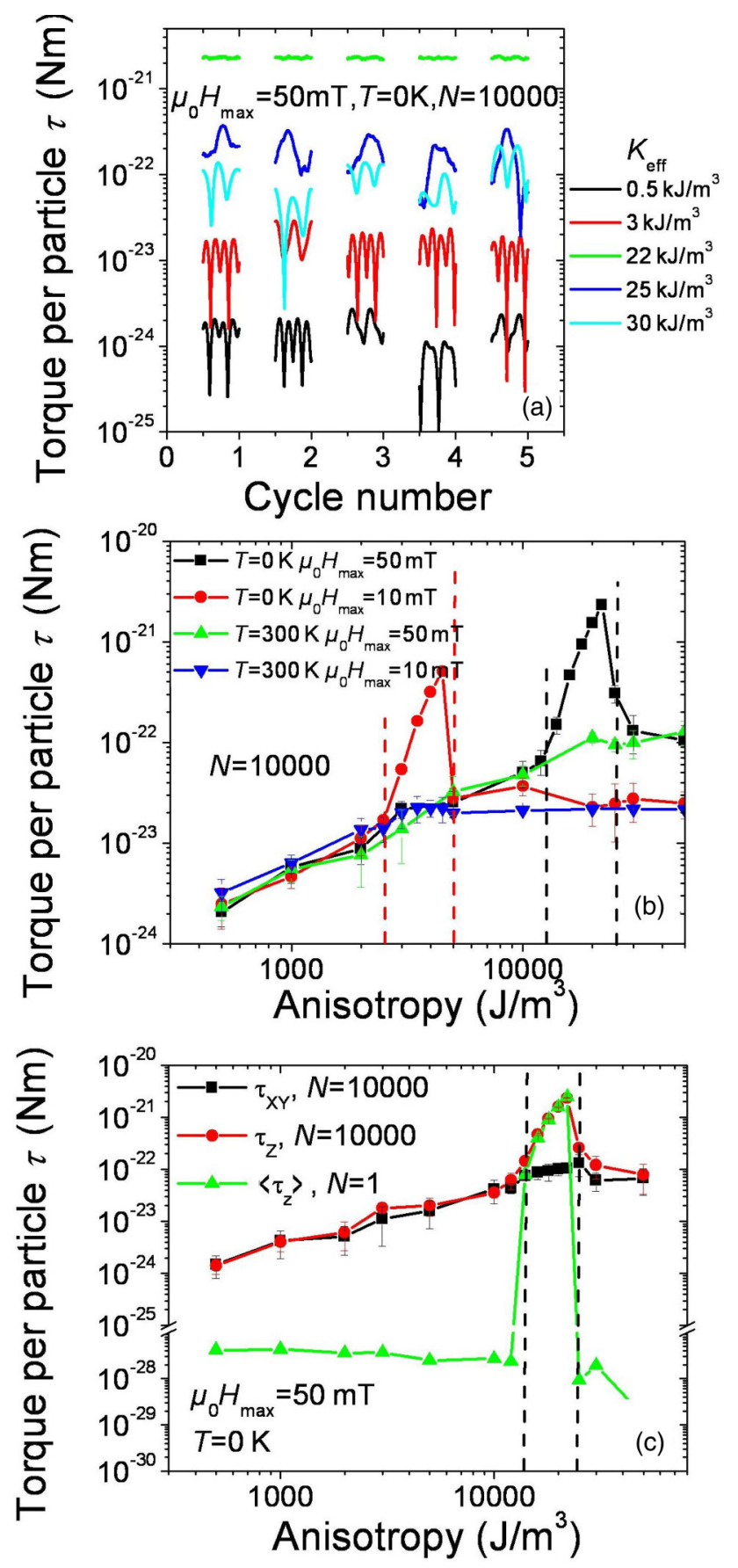

FIG. 9. Torque undergone by an assembly of 10000 noninteracting MNPs with randomly oriented anisotropy axes. (a) Typical example of simulation results: $|\tau|$ as a function of $\phi$ is shown for five different easy axis configurations. Data are shown for five values of $K_{\text {eff }}: 0.5,3,22,25$, and $30 \mathrm{~kJ} / \mathrm{m}^{3}$, where $\mu_{0} H_{\max }=50 \mathrm{mT}$ and $T=0 \mathrm{~K}$. (b) Maximum value of the torque as a function of $K_{\text {eff }}$ for superparamagnetic $(T=300 \mathrm{~K})$ and ferromagnetic $(T=0 \mathrm{~K})$ MNPs for $\mu_{0} H_{\max }=10$ and $50 \mathrm{mT}$. (c) Orientation of torque as a function of anisotropy for $\mu_{0} H_{\max }=50 \mathrm{mT}$ and $T=0 \mathrm{~K}$. The maximum of the torque norm in the $X Y$ plane $\left(\tau_{X Y}\right)$ and along the $Z$ axis $\left(\tau_{Z}\right)$ are plotted. For comparison, the mean value of $\tau_{Z}$, labeled $\left\langle\tau_{Z}\right\rangle$, for a single particle with its easy axis along the $X$ axis is shown. It corresponds to the mean value of $\tau_{Z}$ in Fig. 2(d). (b, c) When $N=10000$, each dot corresponds to an average of five simulations with a different random configuration of the easy axis. 
plus a $\sin ^{2}(\phi)$-like function. This behavior resembles that observed on single MNPs [see Fig. 2(d) and Eqs. (6) and (7)]. For $K_{\text {eff }}=22 \mathrm{~kJ} / \mathrm{m}^{3}$, the torque is very large and almost independent of $\phi$. For $K_{\text {eff }}=25 \mathrm{~kJ} / \mathrm{m}^{3}$, it is a constant value plus a $\sin (\phi)$-like function. It will be shown below-when studying the effect of magnetic interactions - that identifying these shapes will be helpful in interpreting simulation data.

Figure 9(b) summarizes the effect of anisotropy on the torque undergone by superparamagnetic and ferromagnetic MNPs for $\mu_{0} H_{\max }=10$ and $50 \mathrm{mT}$. To plot this graph, the maximum of $|\tau|$ was extracted from five $|\tau|(\phi)$ functions corresponding to five different random axis configuration [see Fig. 9(a)]. A data point in Fig. 9(b) represents the mean of these five values and their standard deviation. This graph illustrates a very interesting feature, which is one of the main findings of this paper: in a given anisotropy range, the torque strongly increases, and there is an anisotropy value maximizing the torque. The vertical dashed lines in Fig. 9(b) illustrate that the condition to maximize the torque is quite simple: the torque increases sharply when $\frac{\mu_{0} H_{\max } M_{S}}{2}<K_{\text {eff }}<\mu_{0} H_{\max } M_{S}$ and is maximized when $K_{\text {eff }}$ is just below $\mu_{0} H_{\max } M_{S}$. For $K_{\text {eff }} \geqslant$ $\mu_{0} H_{\max } M_{S}$, the torque undergoes an abrupt drop greater than one order of magnitude. This range of anisotropy corresponds to the range of parameters where time reversal symmetry is broken, as discussed above and in Ref. [17].

At first sight, this result is surprising because it might appear to contradict what has been found for isolated particles, where an increase of anisotropy was never detrimental to the torque amplitude (see Figs. 3 and 4). However, a closer look at Fig. 2(d) permits an understanding of the underlying phenomenon. In this graph, it can be observed that, for high and low anisotropy values, the $\tau_{Z}(\phi)$ function is symmetrical with respect to the abscissa so that the average value of $\tau_{Z}$ during a $360^{\circ}$ rotation of the magnetic field is null or close to zero. Another way to interpret Fig. 2(d) is to imagine an assembly of particles with their easy axis in the $X Y$ plane with evenly spaced angles. Then, the torque per particle undergone by this assembly after a full rotation of the magnetic field in the $X Y$ plane would be the average of $\tau_{Z}$. Again, for high and low anisotropy values, this average torque would be null. However, it can be observed that, for an intermediate range of anisotropy-curves for $K_{\text {eff }}=20$ and $22 \mathrm{~kJ} / \mathrm{m}^{3}$ are good examples-the $\tau_{Z}(\phi)$ function is not symmetrical with respect to the abscissa: the mean torque undergone by a single particle or by an assembly of MNPs with their easy axes evenly spaced in the $X Y$ plane after a full rotation of the field is nonzero. To illustrate this point, the average value of $\tau_{Z}$ as a function of anisotropy was extracted from Fig. 2(d) and plotted in Fig. 9(c), where an increase by several orders of the magnitude of $\left\langle\tau_{Z}\right\rangle$ for $\frac{\mu_{0} H_{\max } M_{S}}{2}<K_{\text {eff }}<\mu_{0} H_{\max } M_{S}$ is evidenced. In Fig. 9(c), the maximum values of the torque in the plane $\left(\tau_{X Y}\right)$ and perpendicular to the plane $\left(\tau_{Z}\right)$ of the magnetic field are plotted as a function of the anisotropy for the assembly of 10000 particles. These data provide information on the orientation of the torque. It can be observed that for low and large values of anisotropy, the torque is mainly isotropic: no special orientation is favored. On the contrary, when $\frac{\mu_{0} H_{\max } M_{S}}{2}<K_{\text {eff }}<\mu_{0} H_{\max } M_{S}$, it can be clearly seen that the large increase of $|\tau|$ is entirely due to an increase of $\tau_{Z}$. As a
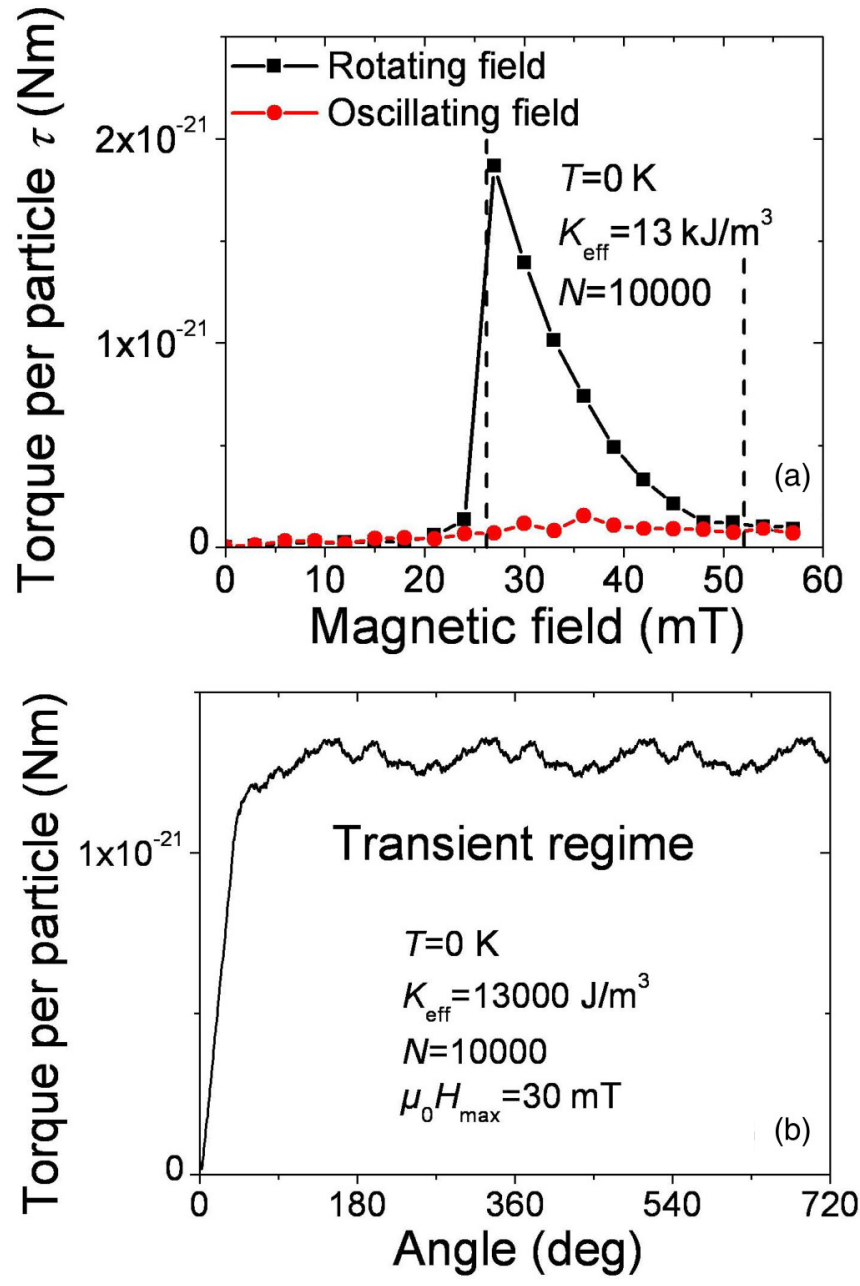

FIG. 10. Torque undergone by an assembly of 10000 noninteracting MNPs with randomly oriented anisotropy axes, $K_{\text {eff }}=13 \mathrm{~kJ} / \mathrm{m}^{3}$, and $T=0 \mathrm{~K}$. (a) Magnetic field dependence of the torque for ( $\mathbf{\square})$ a rotating or $(\bullet)$ an oscillating magnetic field. The pairs of vertical dotted lines correspond to the condition $K_{\text {eff }}=\mu_{0} H_{\max } M_{S}$ and $K_{\text {eff }}=\frac{\mu_{0} H_{\max } M_{S}}{2}$. (b) Angular evolution of the torque during the first rotation of the magnetic field; $\mu_{0} H_{\max }=30 \mathrm{mT}$.

consequence, it is clear from Fig. 9(c) that the rise of torque in the assembly and the increase of $\left\langle\tau_{Z}\right\rangle$ observed in Fig. 2(d) on a single MNP are two consequences of the same phenomenon: they both occur when $\frac{\mu_{0} H_{\max } M_{S}}{2}<K_{\text {eff }}<\mu_{0} H_{\max } M_{S}$, when time reversal symmetry is broken. One should note that, in Fig. 9(c), the perfect quantitative matching between $\left\langle\tau_{Z}\right\rangle$ and $\tau_{Z}$ is completely fortuitous: for a different value of $N$, the maximum value of $\tau_{Z}$ in the assembly could have been above or below the $\left\langle\tau_{Z}\right\rangle$ value for a single MNP (see IV C and Fig. 11 below).

A direct consequence of this finding is that a magnetic field maximizing the torque amplitude for a given anisotropy also exists. This is illustrated in Fig. 10(a), where the magnetic field amplitude of the torque is calculated for $N=10000, K_{\text {eff }}=$ $13 \mathrm{~kJ} / \mathrm{m}^{3}$, and $T=0 \mathrm{~K}$. The torque amplitude is greatly enhanced when $\frac{\mu_{0} H_{K}}{2}<\mu_{0} H_{\max }<\mu_{0} H_{K}$ and is maximized when the magnetic field is just above $\frac{\mu_{0} H_{K}}{2}$. 


\section{B. Rotation vs oscillation, and transient regime}

We have seen just above that the strong increase of torque in a given parameter range is due to a breaking of time reversal symmetry when the magnetic field rotates. From this finding, we can expect that this strong increase does not occur if the magnetic field, instead of rotating, oscillates in a fixed direction. To illustrate this important point, Fig. 10(a) shows the magnetic field dependence of the torque amplitude undergone by an assembly submitted to an oscillating magnetic field. The data are compared with the torque values for an otherwise identical rotating magnetic field. It is very clear from this figure that, when $\frac{\mu_{0} H_{K}}{2}<\mu_{0} H_{\max }<\mu_{0} H_{K}$, the torque generated by an oscillating magnetic field is much lower (up to a factor 30). However, outside this range, the torque undergone by a rotating and an oscillating magnetic field are similar. This again confirms that the breaking of time reversal symmetry due to rotation is absolutely necessary to observe an enhancement of the torque amplitude.

To illustrate further this feature, Fig. 10(b) shows the angular evolution of the torque during the first rotation of the magnetic field. The parameters are the same as those used in Fig. 10(a), and the applied magnetic field equals $30 \mathrm{mT}$, a value for which there is a strong difference in the torque amplitude between an oscillating and a rotating field. We emphasize that such data were not shown in this paper so far: in Fig. 9(a), only the second cycle was shown but not the first. Data in Fig. 10(b) show that the torque evolves from a very small value typical a nonrotating magnetic field to a very large value typical of a rotating magnetic field in less than half of a period, which is thus the duration of the transient regime during which time symmetry is broken and the torque strongly enhanced.

\section{Evolution with the number of particles}

Evolution of the torque amplitude as a function of the number of particles $N$ in the assembly is now presented. It has been calculated for $\mu_{0} H_{\max }=10 \mathrm{mT}, T=0 \mathrm{~K}$, and three representative values of anisotropy: $K_{\text {eff }}=2,4.5$, and $13 \mathrm{~kJ} / \mathrm{m}^{3}$. The middle value corresponds to the anisotropy maximizing the torque for $N=10000$ [see Fig. 9(b)]. The evolution of the torque per particle for $N$ varying between 1 and 1024 is shown in Fig. 11. The torque per particle follows the equation $|\tau|=\tau_{1} N^{-\alpha}$, where $\tau_{1}$ is the torque value of a single particle. For $K_{\text {eff }}=2$ and $13 \mathrm{~kJ} / \mathrm{m}^{3}, \alpha=\frac{1}{2}$. The fact that this simple exponent is found can be easily understood. Indeed, at low magnetic field, the torque generated by an assembly of MNPs can be seen as a 3D random walk problem: each MNP contributes to the total torque by a given randomly oriented vector, the total torque being the sum of these vectors. In this case, the total torque for $N$ particles is $\sqrt{N}$ times the torque of one particle randomly oriented. The torque per particle thus decreases as $\frac{1}{\sqrt{N}}$. On the contrary, for $K_{\text {eff }}=4.5 \mathrm{~kJ} / \mathrm{m}^{3}$, the best fit of simulation data leads to $\alpha=0.22$. This means that the total torque scales as $N^{0.78}$ for an optimized anisotropy. In this case, the torque is not randomly oriented in space but larger in the $Z$ direction, leading to a larger total torque. Similar data have been calculated for $\mu_{0} H=50 \mathrm{mT}$ and three anisotropies $\left(K_{\text {eff }}=10,22\right.$, and $\left.50 \mathrm{~kJ} / \mathrm{m}^{3}\right)$, and similar results were obtained (not shown): $\alpha=\frac{1}{2}$ is obtained for $K_{\text {eff }}=10$ and $50 \mathrm{~kJ} / \mathrm{m}^{3}$, and $\alpha=0.21$ for $K_{\text {eff }}=22 \mathrm{~kJ} / \mathrm{m}^{3}$. The case of

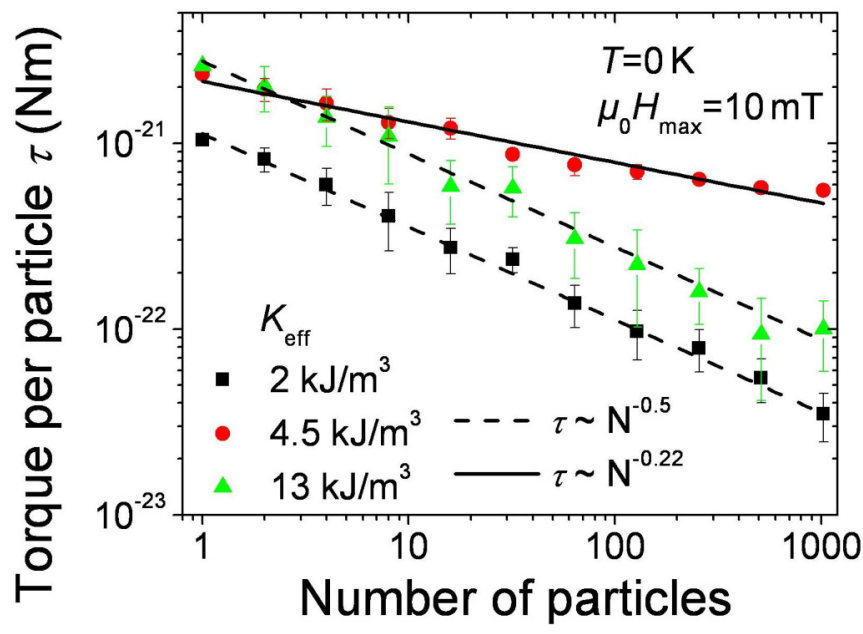

FIG. 11. $|\tau|$ as a function of $N$ for three values of $K_{\text {eff }} ; T=0 \mathrm{~K}$ and $\mu_{0} H_{\max }=10 \mathrm{mT}$. The dashed line corresponds to $\tau \propto \frac{1}{\sqrt{N}}$ and the plain line to $\tau \propto N^{-0.22}$.

superparamagnetic particles has also been tested for several anisotropies at $T=300 \mathrm{~K}$. In this case, the torque decreases with $\alpha=\frac{1}{2}$ for any anisotropy, since there is no increase of the $\tau_{Z}$ component (not shown).

In conclusion, increasing the number of particles in an assembly increases the total torque but decreases the torque per particle and thus the overall energy transferred from the external magnetic field to the system. A good way to avoid this would be to have assemblies of MNPs with perfectly oriented easy axes, so that the total torque is $N$ times the torque per particle $(\alpha=0)$. However, this might be hardly feasible in biological applications. On one other hand, it is shown here that, by choosing an appropriate match between anisotropy and the applied magnetic field, the total torque undergone by the assembly can be greatly enhanced.

We also would like to make clear what happens when several assemblies are measured. For instance, let us imagine that 10 assemblies, each composed of 100 particles with randomly oriented axes, are grafted at different places of a cell membrane. Their anisotropy is such that the equation for the torque per particle $|\tau|=\tau_{1} N^{-0.5}$ is verified. Locally, each assembly will apply a total torque to the membrane of $10 \tau_{1}$ (the individual torque per particle being $0.1 \tau_{1}$ ). Now, if the torque applied to the whole cell is calculated, it is the one generated by an assembly of 1000 particles, i.e., approximately $31 \tau_{1}$. Therefore, a $10 \tau_{1}$ torque would be applied locally by each assembly and would tend to bend the membrane, and a $31 \tau_{1}$ torque would be applied globally and would tend to rotate the whole cell.

\section{Influence of the diameter}

To illustrate the progressive transition from superparamagnetic to ferromagnetic NPs, simulations as a function of the magnetic field amplitude were performed for diameters varying between 20 and $50 \mathrm{~nm}$ at $T=300 \mathrm{~K}$ [see Fig. 12(a)]. This graph is of interest for applications, since it is the calculation of the behavior of an assembly of randomly oriented magnetite NPs as a function of their diameter. One should note that, in Fig. 12(a), $\frac{|\tau|}{\tau_{\max }}$ is plotted to evidence 

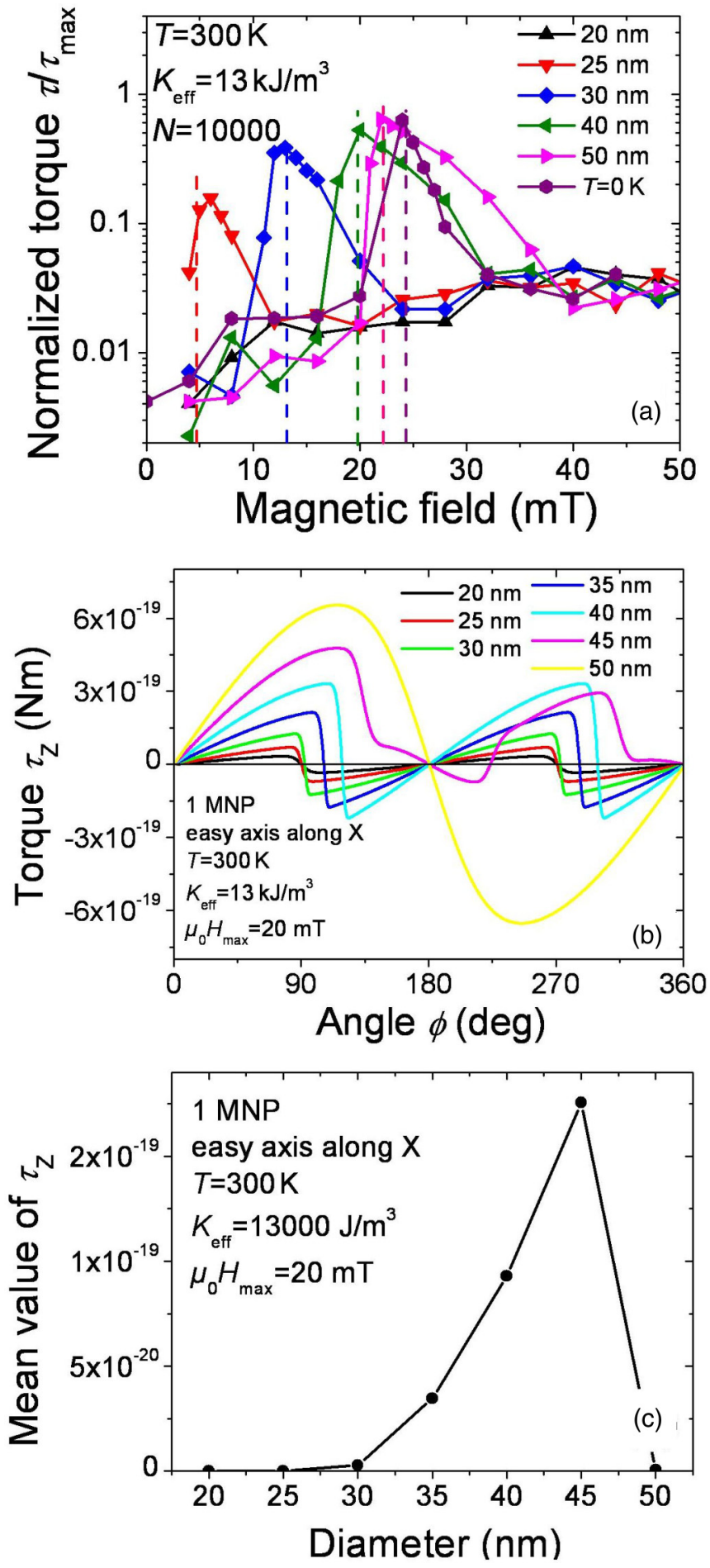

FIG. 12. (a) Normalized torque $\frac{|\tau|}{\tau_{\max }}$ is plotted as a function of $\mu_{0} H_{\max }$ for an assembly of 10000 noninteracting MNPs with randomly oriented anisotropy axes, with $d$ varying from 20 to $50 \mathrm{~nm}$ at $T=300 \mathrm{~K}$. The round dots display the $T=0 \mathrm{~K}$ result for 70-nm MNPs. The vertical dashed lines show the $\mu_{0} H_{\max }$ values maximizing the torque calculated using Eq. (20). (b) $\tau_{Z}(\phi)$ function for a single MNP with its easy axis aligned along the $X$ direction, with $\mu_{0} H_{\max }=20 \mathrm{mT}$ and $T=300 \mathrm{~K}$. The function is averaged over 50000 cycles. (c) Average value of $\tau_{Z}$ extracted from (b), plotted as a function of the diameter. the influence of thermal activation only but that the torque amplitude increases with the diameter because of the increase of $\tau_{\max }$. In this figure, it can be seen that there is still a range of magnetic fields where the torque is greatly enhanced. However, this range now depends on the diameter: for smaller diameter, the magnetic field required to maximize the torque is smaller. One could hypothesize that this increase is due to the increase of the $Z$ component of the torque, similar to what was observed at $T=0 \mathrm{~K}$ [see Fig. 9(c)]. To check this hypothesis, the $\tau_{Z}(\phi)$ function was calculated for a single MNP, with an easy axis along $X$ and a given magnetic field $\mu_{0} H_{\max }=20 \mathrm{mT}$, as a function of the diameter [see Fig. 12(b)]. The mean value of $\tau_{Z}$ is shown as a function of the diameter in Fig. 12(c). There is a very strong relationship between the three graphs in Fig. 12: in Fig. 12(a), for $20 \mathrm{mT}$, the torque is strongly enhanced for diameters in the range $30-50 \mathrm{~nm}$; it is also the range for which $\left\langle\tau_{Z}\right\rangle$ increases for a single particle [see Fig. 12(c)] and for which time reversal symmetry is broken [see Fig. 12(b)].

The fact that the magnetic field maximizing the torque is smaller for a smaller diameter can be understood qualitatively. We have seen above that at $T=0 \mathrm{~K}$, the torque is maximized when the magnetization makes a large jump during the rotation of the magnetic field. For a smaller diameter, this event occurs for a smaller magnetic field, due to the influence of thermal activation, which facilitates the jump. To check the validity of this hypothesis, the magnetic field for which $n \approx 1$ was calculated using Eq. (19), leading to the equation

$$
\mu_{0} H_{\max }=\frac{K_{\text {eff }} V+k_{B} T \ln \left(\frac{f}{v_{0}}\right)}{1.07 M_{S} V} .
$$

The results are shown in Fig. 12(a) as vertical dashed lines, evidencing the very good agreement between the simulations and Eq. (20).

\section{INFLUENCE OF MAGNETIC INTERACTIONS}

The influence of magnetic interactions on the torque undergone by an assembly of MNPs is now presented. To avoid an unnecessarily long presentation, the focus is directly on the experimentally relevant case of an assembly with randomly oriented anisotropy axes. The system under study is a sphere containing $N=10000$ particles; the volume concentration of the particles inside the sphere is $c$. Technically, to generate the position of the MNPs inside the sphere, they are first placed on a cubic lattice and then displaced by a random value in a random direction. The maximum value of the random displacement is chosen so the MNPs cannot overlap with their neighbors. As a consequence, the system under study is a disordered assembly of MNPs. In a typical simulation, the torque as a function of $\phi$ is calculated and the maximum value of $|\tau|$ extracted. The dimensionless concentration, defined as $\frac{\mu_{0} M_{S}^{2} c}{K_{\text {eff }}}$, is introduced. This parameter, representing the balance between the MNP anisotropy and magnetic interactions, often has been found to be important when dealing with an assembly of interacting MNPs [24,26,27]. 


\section{A. Ferromagnetic particles in interaction}

In Fig. 13, the properties of ferromagnetic NPs $(T=0 \mathrm{~K})$ are shown. It is interesting to present them by separating three regimes depending on the anisotropy values: null anisotropy $\left(K_{\text {eff }}=0 \mathrm{~J} / \mathrm{m}^{3}\right)$, strong anisotropy $\left(K_{\text {eff }} \geqslant \mu_{0} H_{\max } M_{S}\right)$, and low anisotropy $\left(K_{\text {eff }} \leqslant \mu_{0} H_{\text {max }} M_{S}\right)$. Results on these three regimes are shown in Figs. 13(a), 13(b), and 13(c), respectively.

Figure 13(a) illustrates the case of MNPs without anisotropy. In the absence of magnetic interaction, the torque is null but increases with magnetic interactions, displays a maximum, and then decreases. This behavior can be understood qualitatively in this way: (i) one first well-known effect of magnetic interactions is to increase the effective anisotropy of MNPs [28], which should in turn increase the total torque of the assembly. (ii) A second effect is to decrease the total magnetization of the assembly, because the MNPs tend to form flux-closure configurations. We have checked this second effect by analyzing data shown in Fig. 13(a): indeed, the magnetization of the assembly decreases continuously with $c$ (not shown). (iii) The combination of these two phenomena with opposite effects leads to the bell-shaped curves displayed in Fig. 13(a). Quantitatively, by varying $M_{S}, \mu_{0} H_{\max }$, and $d$, it has been found that the value at which the torque is maximal depends on the $\frac{c M_{S}}{H_{\max }}$ ratio only. The maximum occurs when $\frac{c M_{S}}{H_{\max }} \approx 1$, as shown in Fig. 13(a). The numerator of this ratio represents physically the average volume magnetization of the sphere full of MNPs when they are saturated. So far, we do not have a simple explanation to propose to the fact that this dimensionless parameter is the one governing the decrease of the torque for null anisotropy MNPs. It can also be observed on this figure that the normalized torque $\frac{|\tau|}{\tau_{\max }}$ slightly decreases with $\mu_{0} H_{\max }$, but the nonnormalized torque $|\tau|$ increases with $\mu_{0} H_{\max }$ (not shown).

Figure 13(b) illustrates the most interesting regime, i.e. where $K_{\text {eff }} \leqslant \mu_{0} H_{\text {max }} M_{S}$. Recall that, for independent MNPs, the torque strongly increases when $\frac{\mu_{0} H_{\max } M_{S}}{2}<$ $K_{\text {eff }}<\mu_{0} H_{\text {max }} M_{S}$ and is maximized for $K_{\text {eff }}$ just below $\mu_{0} H_{\max } M_{S}$ [see Fig. 9(b)]. With the parameters of Fig. 13(b), $\mu_{0} H_{\max } M_{S}=5 \mathrm{~kJ} / \mathrm{m}^{3}$ so that, in Fig. 13(b), MNPs displaying the largest torque are those with $K_{\text {eff }}=4 \mathrm{~kJ} / \mathrm{m}^{3}$. Magnetic interactions for these already optimized MNPs reduce the torque. However, when the anisotropy is smaller than the optimal anisotropy, magnetic interactions increase the torque. In all cases, torque decreases for the largest values of $c$. This figure can thus be explained with arguments similar to those cited above for the null anisotropy regime: a moderate amount of magnetic interactions increases the anisotropy of MNPs so that MNPs with an anisotropy below the optimum see their torque increased. For a large amount of magnetic interactions, the drop of magnetization of the assembly or the fact that the optimal anisotropy is exceeded decreases the torque.

For MNPs with an anisotropy larger than the optimum, shown in Fig. 13(c), the presence of magnetic interactions cannot bring them closer to optimum: the torque only decreases monotonously with increasing concentration, and its amplitude depends on the dimensionless concentration only. At low concentration, $\frac{|\tau|}{\tau_{\max }}=\frac{1}{\sqrt{N}}$ and decreases progressively when
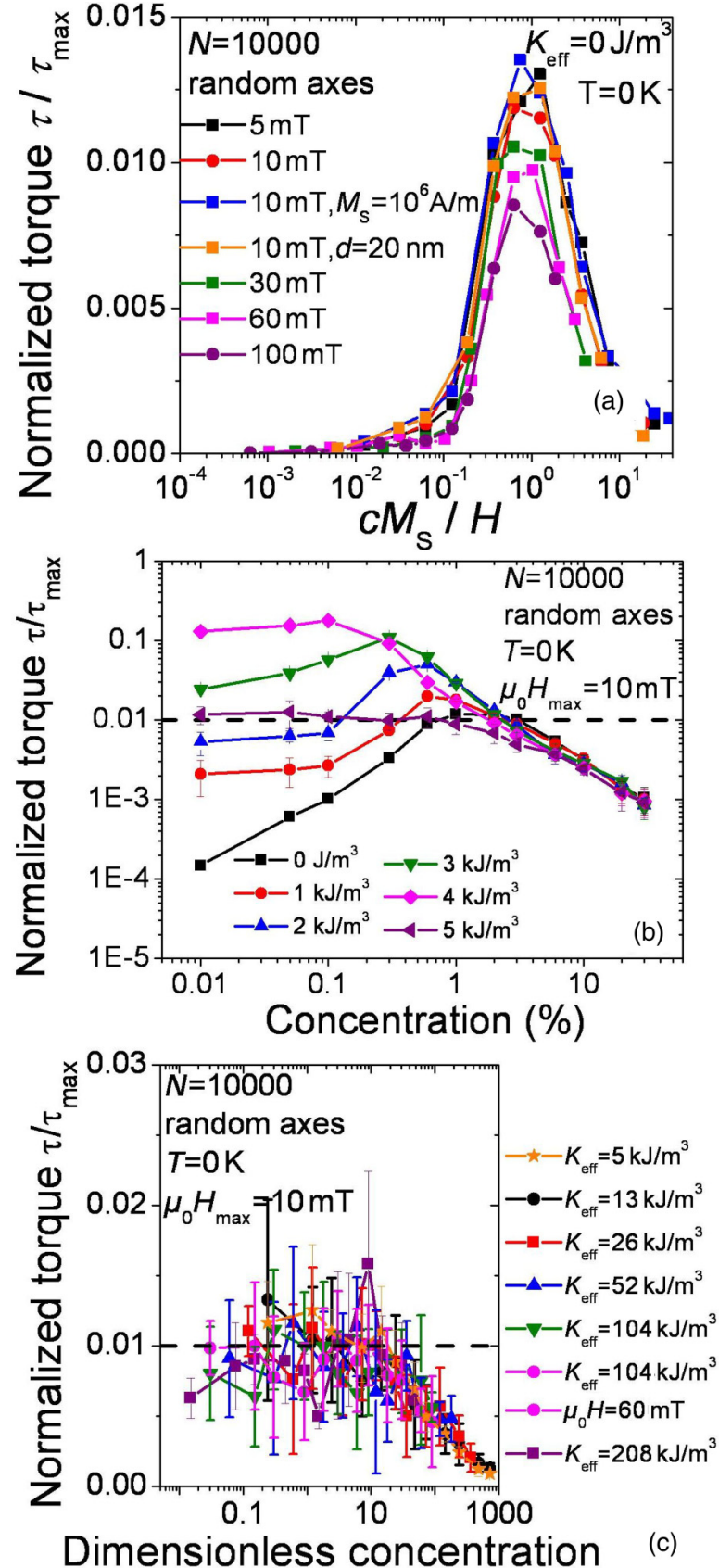

FIG. 13. Influence of volume concentration $c$ on the maximum value of the normalized torque per particle $\frac{|\tau|}{\tau_{\max }}$ undergone in an assembly of $N=10000$ ferromagnetic particles $(T=0 \mathrm{~K})$ in magnetic interaction. The anisotropy axes are randomly oriented. Calculations have been performed for $c=0.01 \%, 0.05 \%, 0.1 \%, 0.3 \%, 0.6 \%, 1 \%$, $2 \%, 3 \%, 6 \%, 10 \%, 20 \%$, and $30 \%$. Unless otherwise specified in the legend, $d=10 \mathrm{~nm}, \mu_{0} H_{\max }=10 \mathrm{mT}$, and $M_{S}=0.5 \times 10^{6} \mathrm{~A} / \mathrm{m}$. (a) Null anisotropy study $\left(K_{\text {eff }}=0 \mathrm{~J} / \mathrm{m}^{3}\right) \cdot \frac{|\tau|}{\tau_{\max }}$ is plotted as a function of $\frac{c M_{S}}{H_{\max }}$ for various values of $\mu_{0} H_{\max }, M_{S}$, and $d$. (b) Low anisotropy study: $K_{\text {eff }} \leqslant \mu_{0} H_{\max } M_{S}\left(5 \mathrm{~kJ} / \mathrm{m}^{3}\right) \cdot \frac{|\tau|}{\tau_{\max }}$ is plotted as a function of $c$ for various values of $K_{\text {eff }}$. (b, c) The horizontal dashed line represents the function $\frac{|\tau|}{\tau_{\max }}=\frac{1}{\sqrt{N}}$. (c) High anisotropy study: $K_{\text {eff }} \geqslant \mu_{0} H_{\max } M_{S}\left(5 \mathrm{~kJ} / \mathrm{m}^{3}\right)$. $\frac{|\tau|}{\tau_{\max }}$ is plotted as a function of the dimensionless concentration $\frac{\mu_{0} M_{S}^{2} c}{K_{\mathrm{eff}}}$ for various values of $K_{\mathrm{eff}}$ and $\mu_{0} H_{\max }$. 
the dimensionless concentration exceeds approximately 10 , vanishing toward zero at large concentrations.

\section{B. Superparamagnetic particles in interaction}

The behavior of superparamagnetic NPs is now presented, starting with a detailed study of an example. In Fig. 14(a), the comparison between the evolution of the normalized torque $\frac{|\tau|}{\tau_{\max }}$ as a function of the concentration for an assembly of ferromagnetic $(T=0 \mathrm{~K})$ and superparamagnetic $(T=300 \mathrm{~K})$ is shown, the other parameters being equal $\left(K_{\text {eff }}=13 \mathrm{~kJ} / \mathrm{m}^{3}, \mu_{0} H_{\max }=10 \mathrm{mT}, d=10 \mathrm{~nm}\right)$. The case of ferromagnetic particles has been studied just above: in this regime, the torque is constant at low concentration and then decreases when $c>1 \%$. In superparamagnetic particles, the torque is more or less constant up to $c=1 \%$ and then abruptly increases. Interestingly, its value for $c=3 \%$ is approximately four times larger than that for ferromagnetic NPs. For larger values of $c$, the torque decreases sharply and becomes similar to the torque of ferromagnetic NPs for $c \approx 10 \%$. Typical shapes for the $|\tau|(\phi)$ function for $c$ values of interest are displayed in Figs. 14(b)-14(d). It can be observed in Fig. 14(c) that, when the concentration is such that the torque of superparamagnetic NPs is maximized, their $|\tau|(\phi)$ function is similar to that observed for noninteracting ferromagnetic NPs with an optimized anisotropy [see Fig. 9(a) for $K_{\text {eff }}=25 \mathrm{~kJ} / \mathrm{m}^{3}$ ]. For a larger concentration, their $|\tau|(\phi)$ function is similar to that of interacting ferromagnetic NPs [see Figs. 14(b) and 14(d)].

At first sight, the fact that superparamagnetic NPs present a larger torque than otherwise identical ferromagnetic particles in a given range of parameters could seem counterintuitive. As a matter of fact, data in Fig. 14 are coherent with the fact that superparamagnetic MNPs, when $c$ increases above $1 \%$, become progressively equivalent to ferromagnetic MNPs, but with an anisotropy which is at the beginning smaller than the real value of $13 \mathrm{~kJ} / \mathrm{m}^{3}$. An analysis of these data using the simulations obtained for noninteracting MNPs [see Fig. 9(b)] permits one to quantify this point: when their torque is maximized for $c=3 \%$, the superparamagnetic NPs
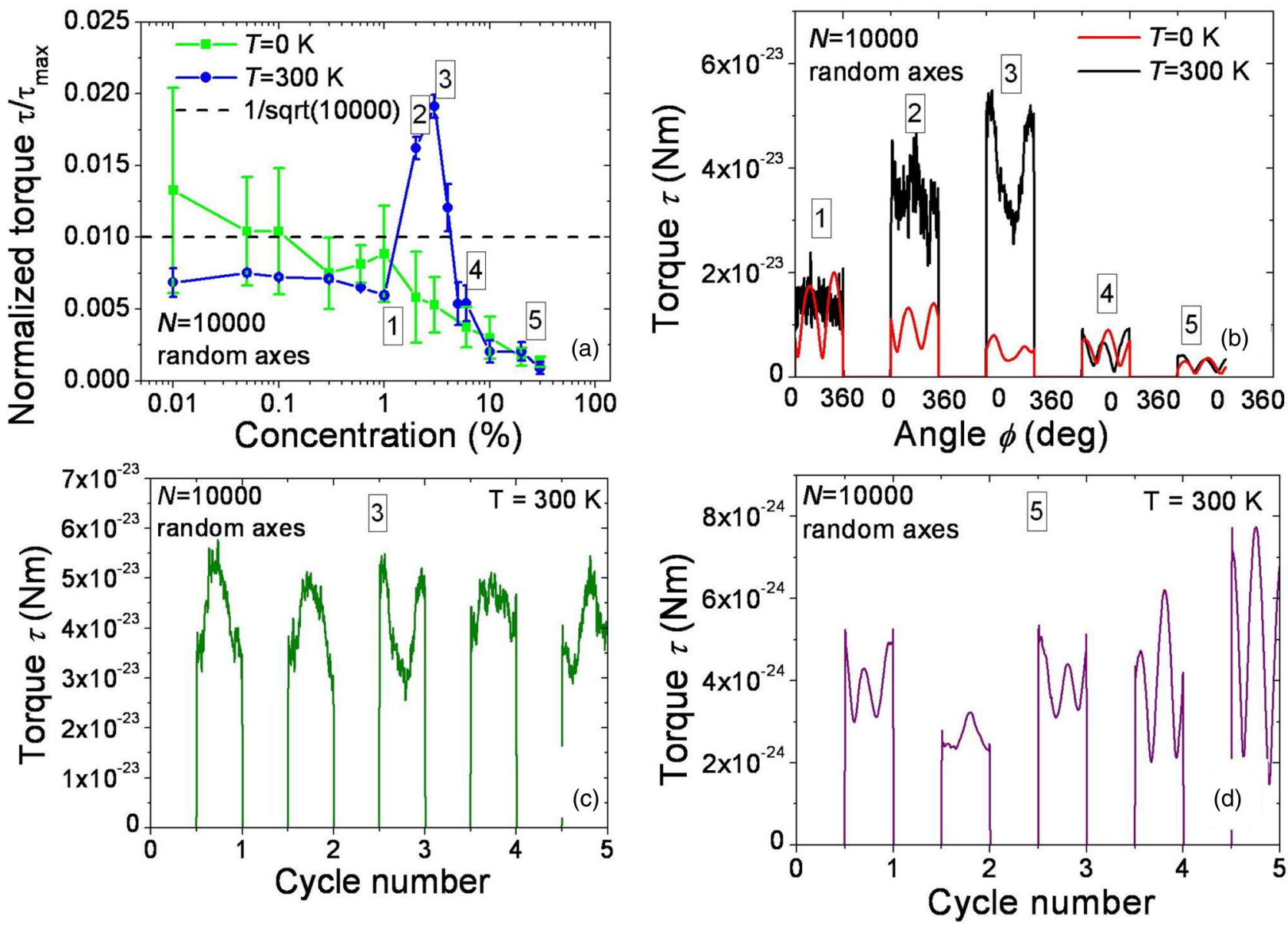

FIG. 14. Influence of volume concentration $c$ on the torque per particle undergone by an assembly of $N=10000$ particles in magnetic interaction; $\mu_{0} H_{\max }=10 \mathrm{mT}, K_{\text {eff }}=13 \mathrm{~kJ} / \mathrm{m}^{3}$, and $d=10 \mathrm{~nm}$. The anisotropy axes are randomly oriented. $c$ values used in the simulations

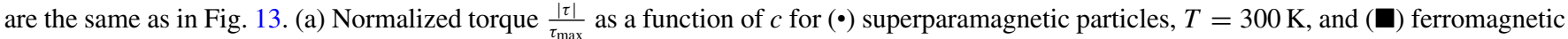
particles, $T=0 \mathrm{~K}$. The labels 1, 2, 3, 4, and 5 represent points of interest corresponding to $c=1 \%, 2 \%, 3 \%, 6 \%$, and $30 \%$. The horizontal dashed line represents the function $\frac{|\tau|}{\tau_{\max }}=\frac{1}{\sqrt{N}}$. (b) Typical evolution of $|\tau|$ with $\phi$ obtained for the points of interests labeled in (a). (c, d) Evolution of $|\tau|$ with $\phi$ for five different configurations of the easy axes at $T=300 \mathrm{~K}$ for (c) $c=3 \%$ and (d) $c=30 \%$. This corresponds to the data points labeled 3 and 5 in (a). (b-d) Data at $300 \mathrm{~K}$ have been smoothed with 50-point averaging to reduce the noise amplitude and enhance the clarity of the figure. 

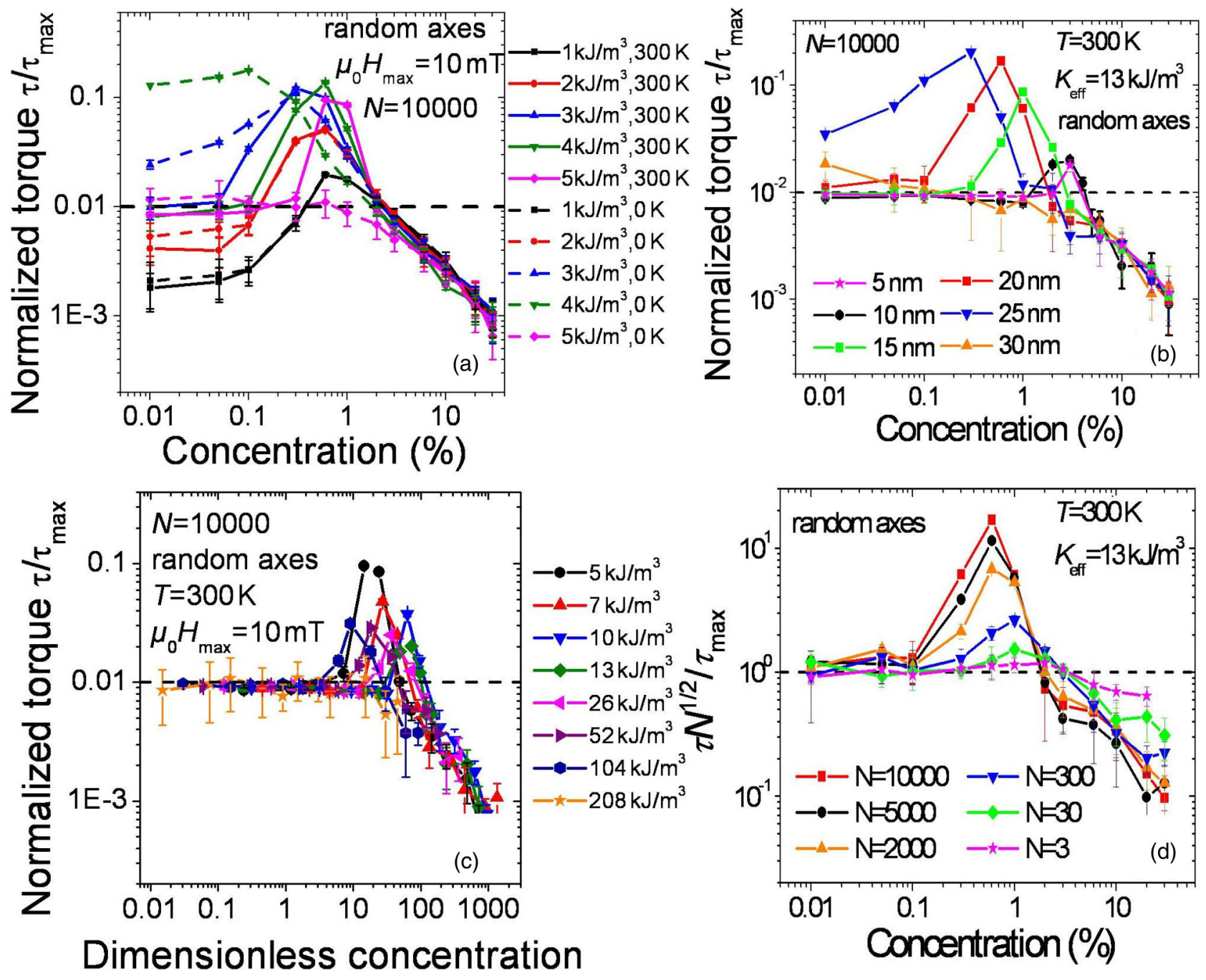

FIG. 15. Influence of $c$ on the torque undergone by an assembly of $N=10000$ interacting superparamagnetic particles with randomly oriented axes; $T=300 \mathrm{~K}$. Unless otherwise specified, $\mu_{0} H_{\max }=10 \mathrm{mT}, K_{\mathrm{eff}}=13 \mathrm{~kJ} / \mathrm{m}^{3}$, and $d=10 \mathrm{~nm}$. (a, c) $\tau / \tau_{\max }$ as a function of concentration or dimensionless concentration for various values of $K_{\text {eff }}$ for the (a) low anisotropy regime, with $K_{\text {eff }}<\mu_{0} H_{\text {max }} M_{S}$ and (c) high anisotropy regimes, with $K_{\text {eff }}>\mu_{0} H_{\max } M_{S}$. (a) Data for $T=0 \mathrm{~K}$ are recalled for comparison. (b) $\tau / \tau_{\max }$ as a function of $c$ for $d$ varying between 5 and $30 \mathrm{~nm}$. (d) $\frac{|\tau| \sqrt{N}}{\tau_{\max }}$ as a function of $c$ for various values of $N$. (a-d) The horizontal dashed line represents (a-c) the function $\frac{|\tau|}{\tau_{\max }}=\frac{1}{\sqrt{N}}(\mathrm{~d})$ the function $\frac{|\tau| \sqrt{N}}{\tau_{\max }}=1$.

in Fig. 14(a) undergo a torque which is equivalent to that of noninteracting ferromagnetic NPs, with a $K_{\text {eff }}$ value in the $3-5 \mathrm{~kJ} / \mathrm{m}^{3}$ range. Then, for a larger value of $c$, the properties are identical to those of ferromagnetic NPs with $K_{\text {eff }}=13 \mathrm{~kJ} / \mathrm{m}^{3}$.

A more systematic study of the properties of superparamagnetic NPs as a function of several parameters $\left(K_{\text {eff }}, d, \mu_{0} H_{\text {max }}\right)$ is shown in Fig. 15. Similar to the case of ferromagnetic NPs presented above, it is convenient to present the data by distinguishing two regimes, depending on whether $K_{\text {eff }}$ is smaller than or greater than $\mu_{0} H_{\max } M_{S}$. Figures 15 (a) and 15 (c) display the data obtained in the low and high anisotropy regime, respectively. In all cases, at $300 \mathrm{~K}$, the MNPs see their torque increased by the presence of magnetic interactions. The only exception is when $K_{\text {eff }}>200 \mathrm{~kJ} / \mathrm{m}^{3}$, but, in this case, the MNPs are not superparamagnetic at $300 \mathrm{~K}$. In the low anisotropy regime, MNPs at $300 \mathrm{~K}$ reach, for a given concentration range, a torque similar to that of equivalent MNPs at $T=0 \mathrm{~K}$. In the high anisotropy regime, there is always a concentration range where superparamagnetic NPs undergo a torque larger - and sometimes much larger, up to one order of magnitude - than equivalent MNPs at $T=0 \mathrm{~K}$. This is equivalent to what was observed in Fig. 14(a) and was qualitatively explained above. For a large degree of interactions, the torque decreases; in the low (high) anisotropy regime, this decrease depends on the concentration (dimensionless concentration) value. This was already the case at $T=0 \mathrm{~K}$ [see Figs. 13(b) and 13(c)].

Figure 15(b) displays the concentration dependence of the torque as a function of the diameter. This figure is very relevant with respect to experiments, because it provides the conditions needed to optimize the torque of magnetite NPs in a small rotating field. These are precisely the conditions which are often used in biology experiments. With the parameter used 
here, MNPs with a diameter of 20-25 $\mathrm{nm}$ are those that permit a maximized normalized torque amplitude. However, a final concentration $c \approx 0.3 \%$ must be targeted to get this large output. In the case where the final concentration is a fixed parameter, this graph illustrates also that there is a concentrationdependant MNP diameter maximizing the torque.

Of course, the strength of magnetic interactions is expected to depend on the size of the assembly. The results presented so far were obtained in an assembly of 10000 MNPs. As an illustration, the influence of $N$ on the torque properties of superparamagnetic NPs is shown in Fig. 15(d). In the ordinate of this graph, $\frac{|\tau| \sqrt{N}}{\tau_{\max }}$ is plotted, so the direct effect of $N$ on the torque per particle is cancelled (see Fig. 11). It illustrates the consequences of the interaction strength attenuation only. A natural consequence of this attenuation is that the maximum of the torque is shifted toward a larger concentration when decreasing $N$ : a larger concentration is required to generate an equivalent dipolar field in an assembly of reduced size. Globally, both the beneficial effect of interactions at moderate concentration and the detrimental effect at large concentration progressively disappear when $N$ decreases, so the torque amplitude becomes progressively concentration independent.

\section{SUMMARY AND CONCLUSION}

Our paper based on kinetic Monte Carlo simulations has permitted us to elucidate several properties of interest on the torque undergone by assemblies of MNPs. Basic properties of isolated ferromagnetic MNPs were first recalled. In this case, as illustrated in Figs. 2 and 3, maximizing the torque only requires maximizing the MNP anisotropy and the magnetic field. The value of the torque then equals the smaller of $K_{\text {eff }} V$ or $\tau_{\max }$, which depends on whether $\mu_{0} H_{\max }$ is greater or smaller than $\frac{K_{\text {eff }}}{M_{S}}$. Although this is strictly true for an easy axis in the plane of the field only, the fact that a single MNP is randomly oriented does not change this result drastically (see Fig. 4). When thermal activation enters into play, its effect is to switch the magnetization at high frequency, so the torque also changes sign at high frequency (see Fig. 6), leading to a decrease of the average torque felt by the MNP. However, it might be interesting not to forget that the instantaneous torque undergone by a superparamagnetic NP is the same as that of an otherwise equivalent ferromagnetic NP (see Fig. 6). An equation that calculates this average torque, as well as its domain of validity, has been proposed and compared with simulations [see Figs. 7 and 8 and Eq. (18)].

The case of an assembly of MNPs is more complex. First, one can note that a reminiscence of single MNP properties appears in the angle dependence of the torque: the $\sin ^{2}(2 \phi)$ or the $\sin ^{2}(\phi)$ signature of the torque in single MNPs is still visible in the angle dependence of the torque of an assembly of 10000 MNPs [see Fig. 9(a)]. Interestingly, simulations clearly show that there is a way to enhance the torque in an assembly of MNPs: the time reversal symmetry must be broken. In this case, the torque undergone by the assembly can become very large due to an increase in the $Z$ component of the torque. This enhancement is possible only if the magnetic field rotates, not if it oscillates [see Fig. 10(a)], and occurs for ferromagnetic particles when the condition $\frac{\mu_{0} H_{\max } M_{S}}{2}<K_{\text {eff }}<\mu_{0} H_{\max } M_{S}$ is fulfilled. For ferromagnetic particles, the torque is maximized when $K_{\text {eff }}$ is just below $\mu_{0} H_{\max } M_{S}$. When thermally activated switches are possible, the torque is maximized when there is one or two switches during the magnetic field rotation. An equation has been proposed which estimates when this occurs, and good agreement with the simulations has been found [see Fig. 12 and Eq. (20)].

In assemblies of $N$ MNPs, in most cases, the total torque increases as $\sqrt{N}$, and the torque per unit volume thus decreases as $\frac{1}{\sqrt{N}}$. However, this rule suffers two noticeable exceptions: (i) if all the easy axes are aligned, the total torque scales as $N$ and the torque per unit volume is constant. This means that, whenever it is possible experimentally, this situation should be favored. For instance, it could be useful to synthesize beads in which MNPs have aligned anisotropy axes. Also, it might be interesting to apply a constant magnetic field during MNP accumulation at the cell membrane or inside cells to favor the alignment of easy axes during biological experiments. (ii) When time reversal symmetry is broken, the total torque grows more rapidly than $\sqrt{N}$; an increase following $N^{0.78}$ was extracted from simulations in optimized conditions for noninteracting ferromagnetic NPs (see Fig. 11).

The presence of magnetic interactions makes the problem more complex. First, the study of ferromagnetic NPs without anisotropy show that magnetic interactions have a tendency to increase anisotropy but also to decrease magnetization of the assembly, giving a bell-shaped dependence to the concentration dependence of the torque [see Fig. 13(a)]. The latter is maximized when $\mu_{0} H_{\max } \approx c \mu_{0} M_{S}$. The decrease in magnetization, and thus of the torque at large concentration, is a general feature observed in all cases.

The fact that the interactions increase anisotropy has a consequence for ferromagnetic MNPs for which $K_{\text {eff }}<$ $\mu_{0} H_{\max } M_{S}$ : magnetic interactions of moderate intensity increase their torque [see Fig. 13(b)]. For ferromagnetic particles with a larger anisotropy, only the decrease of torque at large concentration is observed [see Fig. 13(c)].

Finally, the presence of magnetic interactions on superparamagnetic NPs, which is the most relevant for applications, is very interesting. In almost all cases, interactions strongly increase the torque above the value $\tau=\frac{\tau_{\max }}{\sqrt{N}}$. This means that, for a given concentration, which depends on several parameters ( $d, K_{\text {eff }}, N, \mu_{0} H_{\text {max }}$; see Fig. 15), break of the time reversal symmetry and increase of the $Z$ component of the torque occur. Interestingly, it occurs even for particles with $K_{\text {eff }}>\mu_{0} H M_{S}$, which do not display any breaking of time reversal at $T=0 \mathrm{~K}$ when they are ferromagnetic. Everything happens as if highanisotropy NPs in the superparamagnetic regime, in a given range of concentrations, behave exactly as low-anisotropy, noninteracting ferromagnetic NPs. It is thus possible to enhance greatly the torque generated by superparamagnetic NPs by adjusting their structural characteristics.

In conclusion, the kinetic Monte Carlo simulations that we have developed have permitted us to get an insight into the microscopic phenomena responsible for the torque amplitude in assemblies of MNPs. The problem is much more complex than one would have thought initially, but we have shown evidence of trends and provided analytical formulas that permit calculations when simulations are not available. The concepts 
presented in this article should help chemists and biologists synthesize nano-objects with optimized torque properties. For physicists, it would be interesting to test experimentally the results described in this article. For this purpose, torque measurements on well-characterized assemblies of nanoparticles should be performed and compared with numerical simulations.

\section{ACKNOWLEDGMENTS}

We thank Thomas Blon for his careful reading of the manuscript and fruitful discussions. This work was supported by the ITMO Cancer during the "Plan cancer 2009-2013" and was performed using HPC resources from CALMIP (Grant No. 2015-P1405).
[1] J. Dobson, Nat. Nanotechnol. 3, 139 (2008).

[2] Y. I. Golovin, S. L. Gribanovsky, D. Y. Golovin, N. L. Klyachko, A. G. Majouga, A. Master, M. Sokolsky, and A. V. Kabanov, J. Controlled Release 219, 43 (2015).

[3] E. A. Périgo, G. Hemery, O. Sandre, D. Ortega, E. Garaio, F. Plazaola, and F. J. Teran, Appl. Phys. Rev. 2, 041302 (2015).

[4] S. Carregal-Romero, P. Guardia, X. Yu, R. Hartmann, T. Pellegrino, and W. J. Parak, Nanoscale 7, 570 (2015).

[5] M. Yamaguchi, A. Ito, A. Ono, Y. Kawabe, and M. Kamihira, ACS Synth. Biol. 3, 273 (2014).

[6] H. Huang, S. Delikanli, H. Zeng, D. M. Ferkey, and A. Pralle, Nat. Nanotechnol. 5, 602 (2010).

[7] A. Tay, A. Kunze, C. Murray, and D. Di Carlo, ACS Nano 10, 2331 (2016), and references therein.

[8] D. Kilinc, C. L. Dennis, and G. U. Lee, Adv. Mater. 28, 5672 (2016).

[9] Z. G. Forbes, B. B. Yellen, D. S. Halverson, G. Fridman, K. A. Barbee, and G. Friedman, IEEE Trans. Biomed. Eng. 55, 643 (2008).

[10] F. Mosconi, J. F. Allemand, and V. Croquette, Rev. Sci. Instrum. 82, 034302 (2011).

[11] Y. I. Golovin, N. L. Klyachko, M. Sokolsky-Papkov, and A. V. Kabanov, Bull. Russ. Acad. Sci. Phys. 77, 1350 (2013).

[12] Y. I. Golovin, S. L. Gribanovskii, D. Y. Golovin, N. L. Klyachko, and A. V. Kabanov, Phys. Solid State 56, 1342 (2014).

[13] Y. I. Golovin, S. L. Gribanovskii, N. L. Klyachko, and A. V. Kabanov, Tech. Phys. 59, 932 (2014).

[14] Y. I. Golovin, N. L. Klyachko, D. Y. Golovin, M. V. Efremova, A. A. Samodurov, M. Sokolski-Papkov, and A. V. Kabanov, Tech. Phys. Lett. 39, 240 (2013).
[15] E. Zhang, M. F. Kircher, M. Koch, L. Eliasson, S. N. Goldberg, and E. Renström, ACS Nano 8, 3192 (2014).

[16] S. I. Denisov, T. V. Lyutyy, P. Hänggi, and K. N. Trohidou, Phys. Rev. B 74, 104406 (2006).

[17] M. M. van Oene, L. E. Dickinson, F. Pedaci, M. Köber, D. Dulin, J. Lipfert, and N. H. Dekker, Phys. Rev. Lett. 114, 218301 (2015).

[18] H. Mamiya and B. Jeyadevan, Sci. Rep. 1, 157 (2011).

[19] N. A. Usov and B. Y. Liubimov, J. Appl. Phys. 112, 23901 (2012).

[20] Y. L. Raikher and V. I. Stepanov, J. Magn. Magn. Mater. 320, 2692 (2008).

[21] S. Leulmi, H. Joisten, T. Dietsch, C. Iss, M. Morcrette, S. Auffret, P. Sabon, and B. Dieny, Appl. Phys. Lett. 103, 132412 (2013).

[22] X. J. A. Janssen, A. J. Schellekens, K. van Ommering, L. J. van IJzendoorn, and M. W. J. Prins, Biosens. Bioelectron. 24, 1937 (2009).

[23] J. G. Ovejero, D. Cabrera, J. Carrey, T. Valdivielso, G. Salas, and F. J. Teran, Phys. Chem. Chem. Phys. 18, 10954 (2016).

[24] R. P. Tan, J. Carrey, and M. Respaud, Phys. Rev. B 90, 214421 (2014).

[25] J. Carrey, B. Mehdaoui, and M. Respaud, J. Appl. Phys. 109, 083921 (2011).

[26] C. Martinez-Boubeta, K. Simeonidis, D. Serantes, I. CondeLeborán, I. Kazakis, G. Stefanou, L. Peña, R. Galceran, L. Balcells, C. Monty, D. Baldomir, M. Mitrakas, and M. Angelakeris, Adv. Funct. Mater. 22, 3737 (2012).

[27] R. P. Tan, J. S. Lee, J. U. Cho, S. J. Noh, D. K. Kim, and Y. K. Kim, J. Phys. D 43, 165002 (2010).

[28] F. Luis, F. Petroff, J. M. Torres, L. M. García, J. Bartolomé, J. Carrey, and A. Vaurès, Phys. Rev. Lett. 88, 217205 (2002). 